Research Paper

\title{
A Refined Higher Order Theory for Statics and Dynamics of Doubly Curved Shells
}

\author{
TARUN KANT* and DEVESH PUNERA \\ Department of Civil Engineering, Indian Institute of Technology Bombay, Powai, Mumbai 400 076, \\ India
}

(Received on 14 October 2016; Accepted on 21 November 2016)

\begin{abstract}
A complete formulation of static and dynamic analysis is presented using higher order shear and normal deformation theory (HOSNT) with twelve middle surface displacement parameters for doubly curved shells. Mathematical difficulty of obtaining a three dimensional (3D) solution for problems of plates and shells steered the development of two dimensional (2D) theories. Present model considers transverse shear strains and normal strains thus also incorporating rotary inertia and subsequent higher order expression in dynamic terms. A variational principle based on minimization of energy is used to derive the set of governing differential equations and associated boundary conditions. The theory presented here also uses extended thickness criteria where square of thickness to radius of curvature is considered less than unity instead of the classical assumption of taking thickness to radius of curvature less than unity. Problem of isotropic open cylindrical shell is solved and results are compared with available 3D solutions.
\end{abstract}

Keywords: Higher Order Theory; Doubly Curved Shell; Cylindrical Shell; Static; Dynamic

\section{Introduction}

A structural system bounded by two curved surfaces and separated by a small distance compared to the in-surface dimensions is called a shell structure. The small dimension or thickness, defined in normal direction to surface, is the governing term between the class of thin and thick shells. Thickness of the shell could be constant throughout as well as variable. The fact of relatively small thickness dimension reduces the three-dimensional (3D) shell problem to a two-dimensional (2D) surface with sufficient approximations. Thus middle surface or a reference surface is selected where displacements are defined as the function of shell principal coordinates. If we consider any one co-ordinate parameter (say $\alpha_{2}$ ) to take constant value and second parameter $\left(\alpha_{1}\right.$ for now) to be variable, we obtain family of curves for variable co-ordinate parameter $\alpha_{1}$. It is assumed that two families of coordinate curves are orthogonal to each other and coincident with the directions of principal curvatures (Kraus 1967). Here we define a curvilinear system (Fig. 1) with two orthogonal principle coordinates, i.e., $\left(\alpha_{1}, \alpha_{2}\right)$ along the surface and ' $z$ ' being normal to the surface. $\hat{e}_{1}, \hat{e}_{2}$ and $\hat{n}$ are mutually orthogonal unit vectors in the above curvilinear system, defined at any point such that $\hat{e}_{1}$ and $\hat{e}_{2}$ are tangential to the principle curvilinear coordinates $\alpha_{1}$ and $\alpha_{2}$ while $\hat{n}$ is normal to the surface.

Geometry of a doubly curved shell in curvilinear coordinate system is shown in Fig. 2. Each point on the reference (or middle) surface corresponds to one single pair of values of $\left(\alpha_{1}, \alpha_{2}, 0\right)$. This allows a simple derivation by fundamental equations of the theory of elastic shells. Points existing on the middle surface can be represented in vector form as:

*Authorfor Correspondence: E-mail: tkant@civil.iitb.ac.in 


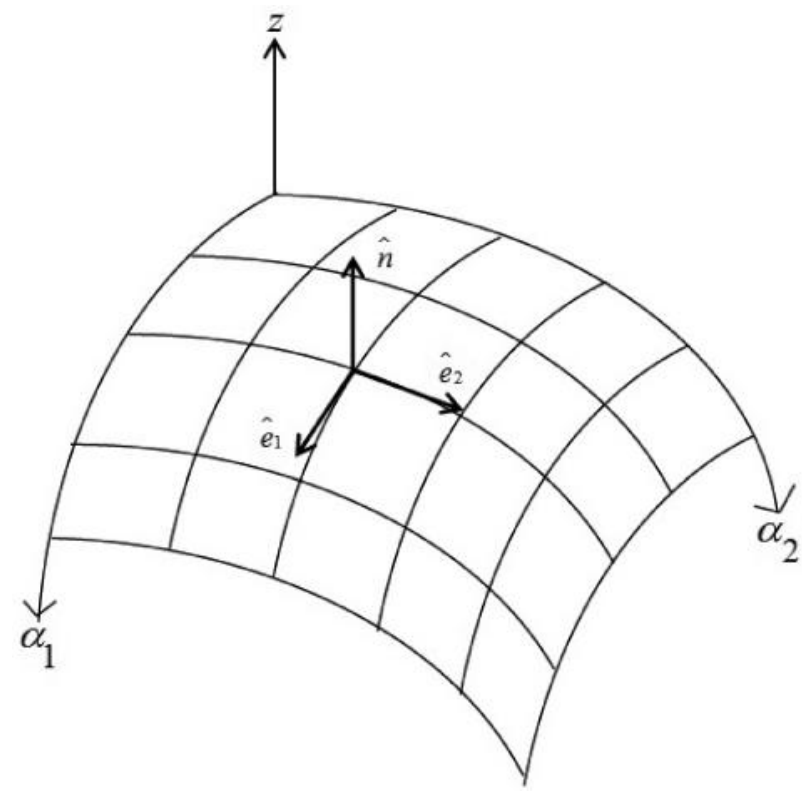

Fig. 1: Curvilinear coordinate system with orthogonal principle coordinates

$$
\boldsymbol{r}=\boldsymbol{r}\left(\alpha_{1}, \alpha_{2}, 0\right)
$$

Any change in the position vector over the middle surface can be given corresponding to the curvilinear coordinate axis. Arbitrary differential line element over curvilinear system between points $\left(\alpha_{1}, \alpha_{2}, 0\right)$ and $\left(\alpha_{1}\right.$ $\left.+d \alpha_{1}, \alpha_{2}+d \alpha_{2}, 0\right)$ can be given as:

$$
d \vec{r}=\frac{\partial \vec{r}}{\partial \alpha_{1}} d \alpha_{1}+\frac{\partial \vec{r}}{\partial \alpha_{2}} d \alpha_{2}
$$

The square of the magnitude of arbitrary line element is defined by scalar product of line vector:

$$
d s_{1}=A_{1} d \alpha_{1} \quad d s_{2}=A_{2} d \alpha_{2}
$$

If only one of the curvilinear coordinate is varied, change in dimension along the two parametric lines $\left(\alpha_{1}, \alpha_{2}\right)$ is given respectively as:

$$
d s_{1}=A_{1} d \alpha_{1}, \quad d s_{2}=A_{2} d \alpha_{2}
$$

Here $d s_{1}$ and $d s_{2}$ are change in curve length along the curvilinear coordinate axis $\alpha_{1}$ and $\alpha_{2}$ corresponding to change in curvilinear coordinates by $d \alpha_{1}$ and $d \alpha_{2}$, respectively. $A_{1}$ and $A_{2}$ are scalar terms called as Lame parameters and are given in terms of Cartesian coordinates $\left(x_{1}, x_{2}, x_{3}\right)$ as:

$$
\begin{aligned}
& A_{1}^{2}=\left|\frac{\partial r}{\partial \alpha_{1}}\right|^{2}=\left(\frac{\partial x_{1}}{\partial \alpha_{1}}\right)^{2}+\left(\frac{\partial x_{2}}{\partial \alpha_{1}}\right)^{2}+\left(\frac{\partial x_{3}}{\partial \alpha_{1}}\right)^{2} \\
& A_{2}^{2}=\left|\frac{\partial r}{\partial \alpha_{2}}\right|^{2}=\left(\frac{\partial x_{1}}{\partial \alpha_{2}}\right)^{2}+\left(\frac{\partial x_{2}}{\partial \alpha_{2}}\right)^{2}+\left(\frac{\partial x_{3}}{\partial \alpha_{2}}\right)^{2}
\end{aligned}
$$

Similarly, position vector of a point existing on a surface parallel to the middle surface at normal distance ' $z$ ' from middle surface is denoted as $\mathbf{R}$ (Fig. $3)$.

$$
\mathbf{R}=\mathbf{r}+z \hat{\mathbf{n}}
$$

Here any change in the position vector between points $\left(\alpha_{1}, \alpha_{2}, z\right)$ and $\left(\alpha_{1}+d \alpha_{1}, \alpha_{2}+d \alpha_{2}, z+d z\right)$ can be given as:

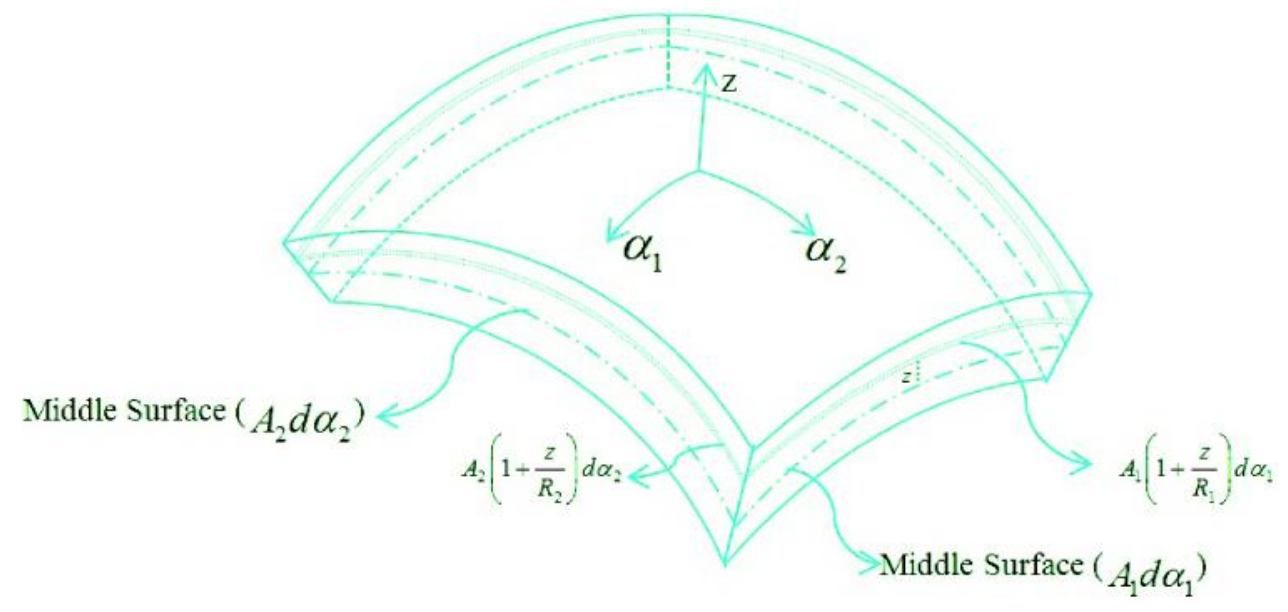

Fig. 2: Geometry of a doubly curved shell 


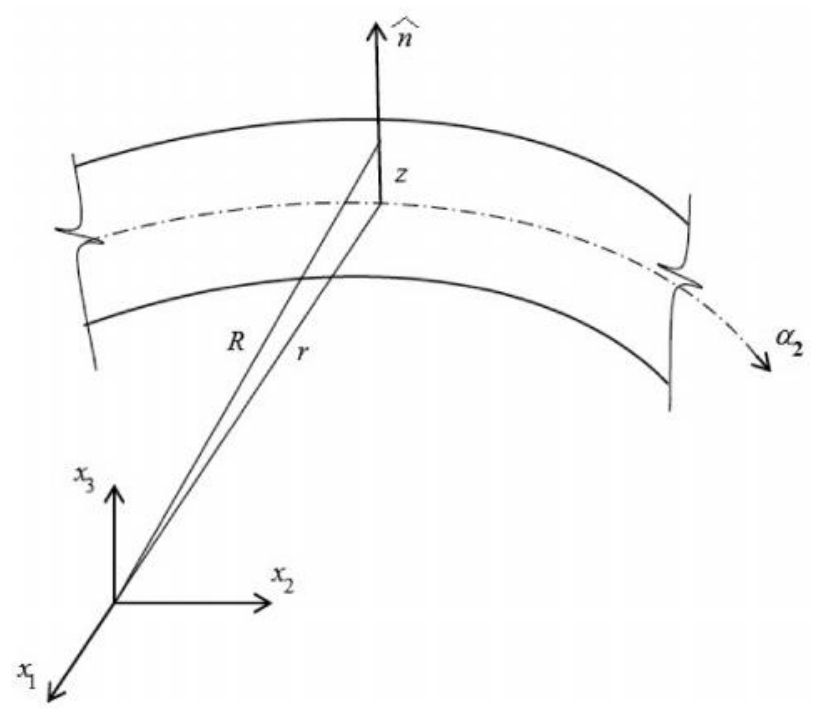

Fig. 3: Through thickness change in curvilinear system

$$
d \vec{R}=\frac{\partial \vec{R}}{\partial \alpha_{1}} d \alpha_{1}+\frac{\partial \vec{R}}{\partial \alpha_{2}} d \alpha_{2}+d z \hat{\mathrm{n}}
$$

Using the Weingarten-Gauss relation, we can further show:

$$
\begin{aligned}
& \frac{\partial \vec{R}}{\partial \alpha_{1}}=\left(1+\frac{z}{R_{1}}\right) \frac{\partial \vec{r}}{\partial \alpha_{1}}, \\
& \frac{\partial \vec{R}}{\partial \alpha_{2}}=\left(1+\frac{z}{R_{2}}\right) \frac{\partial \vec{r}}{\partial \alpha_{2}}
\end{aligned}
$$

So square of the magnitude of line element over a parallel surface ' $z$ ' unit away from middle surface is given as:

$$
\begin{aligned}
(d s(z))^{2}= & d \vec{R} \cdot d \vec{R}=A_{1}^{2}\left(1+\frac{z}{R_{1}}\right)^{2} d \alpha_{1}^{2} \\
& +A_{2}^{2}\left(1+\frac{z}{R_{2}}\right)^{2} d \alpha_{2}^{2}+d z^{2}
\end{aligned}
$$

Changes in length along the parametric lines for any surface at a distance $z$ from the middle surface can be expressed as below :

$$
\begin{aligned}
& d s_{1}(z)=A_{1}\left(1+\frac{z}{R_{1}}\right) d \alpha_{1}, \\
& d s_{2}(z)=A_{2}\left(1+\frac{z}{R_{2}}\right) d \alpha_{2}
\end{aligned}
$$

Love (Love 1888) has presented foundations for the first approximation theories of thin shells. Love shell theory neglects the effect of transverse shear strain, transverse normal strain and normal stress. It assumes thickness to least radius of curvature ratio less than unity. Higher order two-dimensional shell theories include the effect of transverse shear strains and normal strain. Also, Flugge, Lure and Byrne (Kraus 1967) in different articles extended the thickness criteria by assuming square of thickness to least radius of curvature ratio less than unity instead of the above classical assumption. Present formulation gives a higher order shear and normal deformation theory (HOSNT12) with twelve middle surface parameters and also considers Flugge type extended thickness criteria for shells. Present work is extension of the work by Kant (Kant 1976), (Kant 1981b; Kant 1981a), (Kant 2002) with eleven middle surface parameters for doubly curved shells.

\section{Theoretical Formulations}

\section{Displacement Model}

In order to approximate the 3D elasticity problem to two-dimensional problem, displacement parameters are expanded in the form of Taylor's series in terms of thickness coordinate ' $z$ ' about middle surface. Displacement model for present formulation is given as:

$$
\begin{aligned}
u\left(\alpha_{1}, \alpha_{2}, z\right) \approx & u_{0}\left(\alpha_{1}, \alpha_{2}\right)+z \theta_{1}\left(\alpha_{1}, \alpha_{2}\right) \\
& +z^{2} u_{0}^{*}\left(\alpha_{1}, \alpha_{2}\right)+z^{3} \theta_{1}^{*}\left(\alpha_{1}, \alpha_{2}\right) \\
v\left(\alpha_{1}, \alpha_{2}, z\right) \approx & v_{0}\left(\alpha_{1}, \alpha_{2}\right)+z \theta_{2}\left(\alpha_{1}, \alpha_{2}\right) \\
& +z^{2} v_{0}^{*}\left(\alpha_{1}, \alpha_{2}\right)+z^{3} \theta_{2}^{*}\left(\alpha_{1}, \alpha_{2}\right) \\
w\left(\alpha_{1}, \alpha_{2}, z\right) \approx & w_{0}\left(\alpha_{1}, \alpha_{2}\right)+z \theta_{3}\left(\alpha_{1}, \alpha_{2}\right)
\end{aligned}
$$




$$
+z^{2} w_{0}^{*}\left(\alpha_{1}, \alpha_{2}\right)+z^{3} \theta_{3}^{*}\left(\alpha_{1}, \alpha_{2}\right)
$$

\section{Strain-Displacement Relationship}

$$
\begin{array}{ll}
\begin{array}{l}
\text { Based on the assumption of small displacements and } \\
\text { small strains, the strain-displacement relationship in } \\
\text { an orthogonal curvilinear system, shown in Fig. 1, can } \\
\text { be written as (Kraus 1967). }
\end{array} & \varepsilon_{2}=\frac{1}{A_{2}\left[1+\left(z / R_{2}\right)\right]}\left\{\varepsilon_{2}^{0}+z \kappa_{2}+z^{2} \varepsilon_{2}^{*}+z^{3} \kappa_{2}^{*}\right\} \\
\varepsilon_{1}=\frac{1}{A_{1}\left[1+\left(z / R_{1}\right)\right]}\left[\frac{\partial u}{\partial \alpha_{1}}+\frac{v}{A_{2}} \frac{\partial A_{1}}{\partial \alpha_{2}}+\frac{A_{1} w}{R_{1}}\right] & \varepsilon_{3}=\left\{\varepsilon_{3}^{0}+z \kappa_{3}+z^{2} \varepsilon_{3}^{*}+z^{3} \kappa_{3}^{*}\right\} \\
\varepsilon_{2}=\frac{1}{A_{2}\left[1+\left(z / R_{2}\right)\right]}\left[\frac{\partial v}{\partial \alpha_{2}}+\frac{u}{A_{1}} \frac{\partial A_{2}}{\partial \alpha_{1}}+\frac{A_{2} w}{R_{2}}\right] & \gamma_{12}=\frac{1}{A_{1}\left[1+\left(z / R_{1}\right)\right]}\left\{\gamma_{12}^{0}+z \chi_{12}+z^{2} \gamma_{12}^{*}+z^{3} \chi_{12}^{*}\right\} \\
\varepsilon_{3}=\frac{\partial w}{\partial z}, \gamma_{12}=\frac{1}{A_{1}\left[1+\left(z / R_{1}\right)\right]} & +\frac{1}{A_{2}\left[1+\left(z / R_{2}\right)\right]}\left\{\lambda_{12}^{0}+z \kappa_{12}+z^{2} \lambda_{12}^{*}+z^{3} \kappa_{12}^{*}\right\} \\
{\left[\frac{\partial v}{\partial \alpha_{1}}-\frac{u}{A_{2}} \frac{\partial A_{1}}{\partial \alpha_{2}}\right]+\frac{1}{A_{2}\left[1+\left(z / R_{2}\right)\right]}\left[\frac{\partial u}{\partial \alpha_{2}}-\frac{v}{A_{1}} \frac{\partial A_{2}}{\partial \alpha_{1}}\right]} & \gamma_{23}=\frac{1}{A_{1}\left[1+\left(z / R_{2}\right)\right]}\left\{\gamma_{23}^{0}+z \kappa_{23}+z^{2} \gamma_{23}^{*}+z^{3} \kappa_{23}^{*}\right\}
\end{array}
$$$$
\gamma_{13}=\frac{1}{A_{1}\left[1+\left(z / R_{1}\right)\right]}\left(\frac{\partial w}{\partial \alpha_{1}}\right)
$$$$
+A_{1}\left[1+\left(z / R_{1}\right)\right] \frac{\partial}{\partial z}\left[\frac{u}{A_{1}\left[1+\left(z / R_{1}\right)\right]}\right]
$$$$
\gamma_{23}=\frac{1}{A_{2}\left[1+\left(z / R_{2}\right)\right]}\left(\frac{\partial w}{\partial \alpha_{2}}\right)
$$$$
+A_{2}\left[1+\left(z / R_{2}\right)\right] \frac{\partial}{\partial z}\left[\frac{v}{A_{2}\left[1+\left(z / R_{2}\right)\right]}\right]
$$

The Taylor series expansions of the 3D displacements described in HOSNT12 model are substituted in equation (4) to obtain strain displacement relations for the proposed theory. For the convenience of further derivation strain relationship is expressed as below.

$$
\varepsilon_{1}=\frac{1}{A_{1}\left[1+\left(z / R_{1}\right)\right]}\left\{\varepsilon_{1}^{0}+z \kappa_{1}+z^{2} \varepsilon_{1}^{*}+z^{3} \kappa_{1}^{*}\right\}
$$

For theory with $\left(h / R_{\text {min }}\right)<<1$

$$
\begin{aligned}
& \varepsilon_{1}=\frac{1}{A_{1}}\left\{\varepsilon_{1}^{0}+z \kappa_{1}+z^{2} \varepsilon_{1}^{*}+z^{3} \kappa_{1}^{*}\right\} \\
& \varepsilon_{2}=\frac{1}{A_{2}}\left\{\varepsilon_{2}^{0}+z \kappa_{2}+z^{2} \varepsilon_{2}^{*}+z^{3} \kappa_{2}^{*}\right\} \\
& \varepsilon_{3}=\left\{\varepsilon_{3}^{0}+z \kappa_{3}+z^{2} \varepsilon_{3}^{*}+z^{3} \kappa_{3}^{*}\right\} \\
& \gamma_{12}=\frac{1}{A_{1}}\left\{\gamma_{12}^{0}+z \chi_{12}+z^{2} \gamma_{12}^{*}+z^{3} \chi_{12}^{*}\right\} \\
& +\frac{1}{A_{2}}\left\{\lambda_{12}^{0}+z \kappa_{12}+z^{2} \lambda_{12}^{*}+z^{3} \kappa_{12}^{*}\right\} \\
& \gamma_{13}=\frac{1}{A_{1}}\left\{\gamma_{13}^{0}+z \kappa_{13}+z^{2} \gamma_{13}^{*}+z^{3} \kappa_{13}^{*}\right\}
\end{aligned}
$$

For theory with $\left(h / R_{\min }\right)^{2}<<1$ 


$$
\gamma_{23}=\frac{1}{A_{2}}\left\{\varepsilon_{23}^{0}+z \kappa_{23}+z^{2} \varepsilon_{23}^{*}+z^{3} \kappa_{23}^{*}\right\}
$$

Terms defined in above equations (5a) and (5b) are expressed as:

$\varepsilon_{1}^{0}=\frac{\partial u_{0}}{\partial \alpha_{1}}+\frac{v_{0}}{A_{2}} \frac{\partial A_{1}}{\partial \alpha_{2}}+\frac{A_{1} w_{0}}{R_{1}}$

$\varepsilon_{2}^{0}=\frac{\partial v_{0}}{\partial \alpha_{2}}+\frac{u_{0}}{A_{1}} \frac{\partial A_{2}}{\partial \alpha_{1}}+\frac{A_{2} w_{0}}{R_{2}}, \varepsilon_{3}^{0}=\theta_{3}$

$\gamma_{12}^{0}=\left(\frac{\partial v_{0}}{\partial \alpha_{1}}-\frac{u_{0}}{A_{2}} \frac{\partial A_{1}}{\partial \alpha_{2}}\right) \lambda_{12}^{0}=\left(\frac{\partial u_{0}}{\partial \alpha_{2}}-\frac{v_{0}}{A_{1}} \frac{\partial A_{2}}{\partial \alpha_{1}}\right)$

$\gamma_{13}^{0}=\frac{\partial w_{0}}{\partial \alpha_{1}}+A_{1} \theta_{1}-\frac{u_{0} A_{1}}{R_{1}}$

$\gamma_{23}^{0}=\frac{\partial w_{0}}{\partial \alpha_{2}}+A_{2} \theta_{2}-\frac{v_{0} A_{2}}{R_{2}}$

$\kappa_{1}=\frac{\partial \theta_{1}}{\partial \alpha_{1}}+\frac{\theta_{2}}{A_{2}} \frac{\partial A_{1}}{\partial \alpha_{2}}+\frac{A_{1} \theta_{3}}{R_{1}}$,

$\kappa_{2}=\frac{\partial \theta_{2}}{\partial \alpha_{2}}+\frac{\theta_{1}}{A_{1}} \frac{\partial A_{2}}{\partial \alpha_{1}}+\frac{A_{2} \theta_{3}}{R_{2}}, \kappa_{3}=2 w_{0}^{*}$

$\chi_{12}=\frac{\partial \theta_{2}}{\partial \alpha_{1}}-\frac{\theta_{1}}{A_{2}} \frac{\partial A_{1}}{\partial \alpha_{2}} \kappa_{12}=\frac{\partial \theta_{1}}{\partial \alpha_{2}}-\frac{\theta_{2}}{A_{1}} \frac{\partial A_{2}}{\partial \alpha_{1}}$

$\kappa_{13}=\frac{\partial \theta_{3}}{\partial \alpha_{1}}+2\left(A_{1} u_{0}^{*}\right) \kappa_{23}=\frac{\partial \theta_{3}}{\partial \alpha_{2}}+2\left(A_{2} v_{0}^{*}\right)$

$\varepsilon_{1}^{*}=\frac{\partial u_{0}^{*}}{\partial \alpha_{1}}+\frac{v_{0}^{*}}{A_{2}} \frac{\partial A_{1}}{\partial \alpha_{2}}+\frac{A_{1} w_{0}^{*}}{R_{1}}$

$\varepsilon_{2}^{*}=\frac{\partial v_{0}^{*}}{\partial \alpha_{2}}+\frac{u_{0}^{*}}{A_{1}} \frac{\partial A_{2}}{\partial \alpha_{1}}+\frac{A_{2} w_{0}^{*}}{R_{2}}, \varepsilon_{3}^{*}=3 \theta_{3}^{*}$ $\gamma_{12}^{*}=\frac{\partial v_{0}^{*}}{\partial \alpha_{1}}-\frac{u_{0}^{*}}{A_{2}} \frac{\partial A_{1}}{\partial \alpha_{2}} \quad \lambda_{12}^{*}=\frac{\partial u_{0}^{*}}{\partial \alpha_{2}}-\frac{v_{0}^{*}}{A_{1}} \frac{\partial A_{2}}{\partial \alpha_{1}}$

$\gamma_{13}^{*}=\frac{\partial w_{0}^{*}}{\partial \alpha_{1}}+3\left(A_{1} \theta_{1}^{*}\right)+\frac{u_{0}^{*} A_{1}}{R_{1}}$

$\gamma_{23}^{*}=\frac{\partial w_{0}^{*}}{\partial \alpha_{2}}+3\left(A_{2} \theta_{2}^{*}\right)+\frac{v_{0}^{*} A_{2}}{R_{2}}$

$\kappa_{1}^{*}=\frac{\partial \theta_{1}^{*}}{\partial \alpha_{1}}+\frac{\theta_{2}^{*}}{A_{2}} \frac{\partial A_{1}}{\partial \alpha_{2}}+\frac{A_{1} \theta_{3}^{*}}{R_{1}}$

$\kappa_{2}^{*}=\frac{\partial \theta_{2}^{*}}{\partial \alpha_{2}}+\frac{\theta_{1}^{*}}{A_{1}} \frac{\partial A_{2}}{\partial \alpha_{1}}+\frac{A_{2} \theta_{3}^{*}}{R_{2}}, \kappa_{3}^{*}=0$

$\chi_{12}^{*}=\frac{\partial \theta_{2}^{*}}{\partial \alpha_{1}}-\frac{\theta_{1}^{*}}{A_{2}} \frac{\partial A_{1}}{\partial \alpha_{2}} \kappa_{12}^{*}=\frac{\partial \theta_{1}^{*}}{\partial \alpha_{2}}-\frac{\theta_{2}^{*}}{A_{1}} \frac{\partial A_{2}}{\partial \alpha_{1}}$

$\kappa_{13}^{*}=\frac{\partial \theta_{3}^{*}}{\partial \alpha_{1}}+2\left(\frac{\theta_{1}^{*} A_{1}}{R_{1}}\right) \kappa_{23}^{*}=\frac{\partial \theta_{3}^{*}}{\partial \alpha_{2}}+2\left(\frac{\theta_{2}^{*} A_{2}}{R_{2}}\right)$

\section{Variational Principle and System of Equations}

Equations of motion are derived using Hamilton's principle as apart from being simple, it also gives the natural boundary conditions to be used with the given problem. Hamilton's principle in terms of potential energy and kinetic energy $K$ for an elastic shell system, changing its position between two instances of time $\left(t_{0}\right.$ to $\left.t_{1}\right)$, is expressed as:

$$
\delta \int_{t_{0}}^{t_{1}}(\Pi-K) d t=0
$$

To derive the system of equations for static problem, Hamilton's principle is reduced to principle of minimum total potential energy defined as:

$$
\delta(\Pi)=0
$$

Potential Energy $\Pi$ is given as: 


$$
\Pi=U-W_{S}-W_{B}-W_{e 1}-W_{e 2}
$$

Where,

$U=$ Strain Energy stored in the body

$W_{S}=$ External work done by the surface forces

$W_{B}=$ External work done by the body forces

$W_{e 1}=$ External work done because of edge stresses along edge of constant

$W_{e 2}=$ External work done because of edge stresses along edge of constant

Work done by the surface forces is given over the area of element, while work done by Body forces is given over the volume of element. Let usdenote body force as $\mathbf{B}$ while $\mathbf{p}$ denotes the vector of surface load intensities for generalized displacement vector u. Subscripts +, - and Oindicate locations at top, bottom and middle surfaces, respectively. Potential energy can be simplified as:

$$
\begin{aligned}
& \Pi==\frac{1}{2} \int_{V} \varepsilon^{t} c d V-\int_{V} \boldsymbol{u}^{t} \boldsymbol{B} d V- \\
& \left(\int_{A} \boldsymbol{u}_{+}^{t} \boldsymbol{p}_{+} d A-\int_{A} \boldsymbol{u}_{-}^{t} \boldsymbol{p}_{-} d A-\int_{A} \boldsymbol{u}_{0}^{t} \boldsymbol{p}_{0} d A\right)-W_{e 1}-W_{e 2}
\end{aligned}
$$

In which $\alpha$ and $\varepsilon$ are stress and strain vectors, respectively. These are defined as:

$$
\begin{aligned}
& \boldsymbol{\sigma}=\left(\sigma_{1}, \sigma_{2}, \sigma_{3}, \tau_{12}, \tau_{13}, \tau_{23}\right)^{\mathrm{t}}, \\
& \boldsymbol{\varepsilon}=\left(\varepsilon_{1}, \varepsilon_{2}, \varepsilon_{3}, \gamma_{12}, \gamma_{13}, \gamma_{23}\right)^{\mathrm{t}}, \\
& \mathbf{u}=(\mathrm{u}, \mathrm{v}, \mathrm{w})^{\mathrm{t}}, \mathbf{p}=\left(\mathrm{q}_{1}, \mathrm{q}_{2}, \mathrm{q}_{n}\right)^{\mathrm{t}}
\end{aligned}
$$

\section{External Work}

External work done by the surface forces can be written as:
Here $q_{i}$ represents loads acting along the vector coordinate ' $i$ ' and,

$$
\begin{gathered}
U_{i}^{+}=u_{i}+\frac{h}{2} \theta_{i}+\frac{h^{2}}{4} u_{i}^{*}+\frac{h^{3}}{8} \theta_{i}^{*} \\
U_{i}^{-}=u_{i}-\frac{h}{2} \theta_{i}+\frac{h^{2}}{4} u_{i}^{*}-\frac{h^{3}}{8} \theta_{i}^{*} \\
\delta W_{S}=\iint_{\alpha_{1} \alpha_{2}}\left\{\left(q_{1} \delta u_{0}+q_{2} \delta v_{0}+q_{n} \delta w_{0}\right)\right\} A_{1} A_{2} d \alpha_{1} d \alpha_{2} \\
+\int_{\alpha_{1}} \int_{\alpha_{2}}\left\{\left(m_{1} \delta \theta_{1}+m_{2} \delta \theta_{2}+m_{n} \delta \theta_{3}\right)\right\} A_{1} A_{2} d \alpha_{1} d \alpha_{2} \\
+\int_{\alpha_{1}} \int_{\alpha_{2}}\left\{\frac{h^{2}}{4}\left(q_{1} \delta u_{0}^{*}+q_{2} \delta v_{0}^{*}+q_{n} \delta w_{0}^{*}\right)\right\} A_{1} A_{2} d \alpha_{1} d \alpha_{2} \\
+\int_{\alpha_{1}} \int_{\alpha_{2}}\left\{\frac{h^{2}}{4}\left(m_{1} \delta \theta_{1}^{*}+m_{2} \delta \theta_{2}^{*}+m_{n} \delta \theta_{3}^{*}\right)\right\} A_{1} A_{2} d \alpha_{1} d \alpha_{2}
\end{gathered}
$$

where,

$$
\begin{aligned}
& q_{i}=q_{i}^{+}\left(1+\frac{h}{2 R_{1}}\right)\left(1+\frac{h}{2 R_{2}}\right)+q_{i}^{-}\left(1-\frac{h}{2 R_{1}}\right)\left(1-\frac{h}{2 R_{2}}\right) \\
& m_{i}=\frac{h}{2}\left[q_{i}^{+}\left(1+\frac{h}{2 R_{1}}\right)\left(1+\frac{h}{2 R_{2}}\right)-q_{i}^{-}\left(1-\frac{h}{2 R_{1}}\right)\left(1-\frac{h}{2 R_{2}}\right)\right]
\end{aligned}
$$

for $i=1,2, n$

\section{Stress Resultants}

Variation in strain energy can be written as:

$$
\begin{gathered}
\delta U=\iint_{\alpha_{1}} \int_{\alpha_{2}} \int_{z}\left(\sigma_{1} \delta \varepsilon_{1}+\sigma_{2} \delta \varepsilon_{2}+\sigma_{3} \delta \varepsilon_{3}+\tau_{12} \delta \gamma_{12}\right. \\
\left.+\tau_{13} \delta \gamma_{13}+\tau_{23} \delta \gamma_{23}\right) A_{1} A_{2} \\
\left(1+\frac{z}{R_{1}}\right)\left(1+\frac{z}{R_{2}}\right) d \alpha_{1} d \alpha_{2} d z
\end{gathered}
$$

$$
W_{S}=\int_{\alpha_{1}} \int_{\alpha_{2}}\left[\left(q_{1}^{+} U_{1}^{+}+q_{2}^{+} U_{2}^{+}+q_{n}^{+} W^{+}\right)\left(1+\frac{h}{2 R_{1}}\right)\left(1+\frac{h}{2 R_{2}}\right)+\left(q_{1}^{-} U_{1}^{-}+q_{2}^{-} U_{2}^{-}+q_{n}^{-} W^{-}\right)\left(1-\frac{h}{2 R_{1}}\right)\left(1-\frac{h}{2 R_{2}}\right)\right] A_{1} A_{2} d \alpha_{1} d \alpha_{2}
$$


Integral of stresses over the unit length of reference (mid) surface of the orthogonal coordinate edges are represented by stress resultants. Integration of the equation (15) through the thickness of the shell defines stress resultants on reference surface and the 3D nature of equation (15) gets transformed into a 2D form. For a general HOSNT12 theory the following stress resultants will have to be defined.

For theory with $\left(h / R_{\min }\right)^{2}<<1$

$$
\left\{\begin{array}{l}
N_{i} \\
M_{i} \\
N_{i}^{*} \\
M_{i}^{*}
\end{array}\right\}=\int_{-\frac{h}{2}}^{\frac{h}{2}} \sigma_{i}\left(1+\frac{z}{R_{j}}\right)\left\{\begin{array}{l}
1 \\
z \\
z^{2} \\
z^{3}
\end{array}\right\} d z
$$

For $i, j=1,2 \& i \neq j$

$$
\left\{\begin{array}{l}
N_{i j} \\
M_{i j} \\
N_{i j}^{*} \\
M_{i j}^{*}
\end{array}\right\}=\int_{-\frac{h}{2}}^{\frac{h}{2}} \tau_{i j}\left(1+\frac{z}{R_{j}}\right)\left\{\begin{array}{l}
1 \\
z \\
z^{2} \\
z^{3}
\end{array}\right\} d z
$$

For $i, j=1,2 \& i \neq j$

$$
\begin{aligned}
& \left\{\begin{array}{l}
Q_{i} \\
S_{i} \\
Q_{i}^{*} \\
S_{i}^{*}
\end{array}\right\}=\int_{-\frac{h}{2}}^{\frac{h}{2}} \tau_{i n}\left(1+\frac{z}{R_{j}}\right)\left\{\begin{array}{l}
1 \\
z \\
z^{2} \\
z^{3}
\end{array}\right\} d z \\
& \left\{\begin{array}{l}
A \\
B \\
D
\end{array}\right\}=\int_{-\frac{h}{2}}^{\frac{h}{2}} \sigma_{n}\left(1+\frac{z}{R_{1}}\right)\left(1+\frac{z}{R_{2}}\right)\left\{\begin{array}{l}
1 \\
z \\
z^{2}
\end{array}\right\} d z
\end{aligned}
$$

For theory with $\left(h / R_{\text {min }}\right)<<1$

$$
\left\{\begin{array}{l}
N_{i} \\
M_{i} \\
N_{i}^{*} \\
M_{i}^{*}
\end{array}\right\}=\int_{-\frac{h}{2}}^{\frac{h}{2}} \sigma_{i}\left\{\begin{array}{l}
1 \\
z \\
z^{2} \\
z^{3}
\end{array}\right\} d z \text { For } i, j=1,2 \& i \neq j
$$

$$
\left\{\begin{array}{l}
N_{i j} \\
M_{i j} \\
N_{i j}^{*} \\
M_{i j}^{*}
\end{array}\right\}=\int_{-\frac{h}{2}}^{\frac{h}{2}} \tau_{i j}\left\{\begin{array}{l}
1 \\
z \\
z^{2} \\
z^{3}
\end{array}\right\} d z \text { For } i, j=1,2 \& i \neq j,
$$$$
\left\{\begin{array}{l}
Q_{i} \\
S_{i} \\
Q_{i}^{*} \\
S_{i}^{*}
\end{array}\right\}=\int_{-\frac{h}{2}}^{\frac{h}{2}} \tau_{i n}\left\{\begin{array}{l}
1 \\
z \\
z^{2} \\
z^{3}
\end{array}\right\} d z \quad \text { For } i=1,2
$$

$$
\left\{\begin{array}{l}
A \\
B \\
D
\end{array}\right\}=\int_{-\frac{h}{2}}^{\frac{h}{2}} \sigma_{n}\left\{\begin{array}{l}
1 \\
z \\
z^{2}
\end{array}\right\} d z
$$

\section{Edge Stresses}

Work done by the edge stresses can be represented on shell cross-section as:

$$
\begin{aligned}
& W_{e 1}=\iint_{\alpha_{2}}\left(\overline{\sigma_{1}} u+\overline{\tau_{12}} v+\overline{\tau_{13}} w\right) A_{2}\left(1+\frac{z}{R_{2}}\right) d \alpha_{2} d z \\
& W_{e 2}=\int_{\alpha_{1}} \int_{z}\left(\overline{\tau_{21}} u+\overline{\sigma_{2}} v+\overline{\tau_{23}} w\right) A_{1}\left(1+\frac{z}{R_{1}}\right) d \alpha_{1} d z
\end{aligned}
$$

Integrating the edge stresses over shell thickness, we get edge stress resultants in equation (17). This reduces 2D expression to $1 \mathrm{D}$ system on edges of reference surface. 


$$
\begin{aligned}
& \delta W_{e 1}=\int_{\alpha_{2}}\left(\overline{N_{1}} \delta u_{0}+\overline{M_{1}} \delta \theta_{1}+\overline{N_{1}^{*}} \delta u_{0}^{*}+\overline{M_{1}^{*}} \delta \theta_{1}^{*}\right) A_{2} d \alpha_{2}+\int_{\alpha_{2}}\left(\overline{N_{12}} \delta v_{0}+\overline{M_{12}} \delta \theta_{2}+\overline{N_{12}^{*}} \delta v_{0}^{*}+\overline{M_{12}^{*}} \delta \theta_{2}^{*}\right) A_{2} d \alpha_{2} \\
& \quad+\int_{\alpha_{2}}\left(\overline{Q_{1}} \delta w_{0}+\overline{S_{1}} \delta \theta_{3}+\overline{Q_{1}^{*}} \delta w_{0}^{*}+\overline{S_{1}^{*}} \delta \theta_{3}^{*}\right) A_{2} d \alpha_{2} \\
& \delta W_{e 2}=\int_{\alpha_{1}}\left(\overline{N_{21}} \delta u_{0}+\overline{M_{21}} \delta \theta_{1}+\overline{N_{21}^{*}} \delta u_{0}^{*}+\overline{M_{21}^{*}} \delta \theta_{1}^{*}\right) A_{1} d \alpha_{1}+\int_{\alpha_{1}}\left(\overline{N_{2}} \delta v_{0}+\overline{M_{2}} \delta \theta_{2}+\overline{N_{2}^{*}} \delta v_{0}^{*}+\overline{M_{2}^{*}} \delta \theta_{2}^{*}\right) A_{1} d \alpha_{1} \\
& \mid+\int_{\alpha_{1}}\left(\overline{Q_{2}} \delta w_{0}+\overline{S_{2}} \delta \theta_{3}+\overline{Q_{2}^{*}} \delta w_{0}^{*}+\overline{S_{2}^{*}} \delta \theta_{3}^{*}\right) A_{1} d \alpha_{1}
\end{aligned}
$$

\section{Strain Energy}

Strain energy expression for the system can be given as:

$$
\begin{aligned}
& \delta U=\iint_{\alpha_{1}} \int_{\alpha_{2}}\left(\sigma_{z} \delta \varepsilon_{1}+\sigma_{2} \delta \varepsilon_{2}+\sigma_{3} \delta \varepsilon_{3}\right. \\
& \left.+\tau_{12} \delta \gamma_{12}+\tau_{13} \delta \gamma_{13}+\tau_{23} \delta \gamma_{23}\right) \\
& A_{1} A_{2}\left(1+\frac{z}{R_{1}}\right)\left(1+\frac{z}{R_{2}}\right) d \alpha_{1} d \alpha_{2} d z \\
& \text { Now } \delta U_{1}=\iint_{\alpha_{1}} \int_{\alpha_{2}} \int_{z}\left(\sigma_{1} \delta \varepsilon_{1}\right) A_{1} A_{2} \\
& \left(1+\frac{z}{R_{1}}\right)\left(1+\frac{z}{R_{2}}\right) d \alpha_{1} d \alpha_{2} d z
\end{aligned}
$$

As we know:

$$
\varepsilon_{1}=\frac{1}{A_{1}\left(1+\left(z / R_{1}\right)\right)}\left\{\varepsilon_{1}^{0}+z \kappa_{1}+z^{2} \varepsilon_{1}^{*}+z^{3} \kappa_{1}^{*}\right\}
$$

So,

$$
\delta U_{1}=\iint_{\alpha_{1}} \int_{\alpha_{2}}\left(\sigma_{z}\left(\delta \varepsilon_{1}^{0}+z \delta \kappa_{1}+z^{2} \delta \varepsilon_{1}^{*}+z^{3} \delta \kappa_{1}^{*}\right)\right) A_{2} \quad+\iint_{\alpha_{1} \alpha_{2}} N_{2}^{*} A_{1}\left(\frac{\partial \delta v_{0}^{*}}{\partial \alpha_{2}}+\frac{\delta u_{0}^{*}}{A_{1}} \frac{\partial A_{2}}{\partial \alpha_{1}}+\frac{A_{2} \delta w_{0}^{*}}{R_{2}}\right) d \alpha_{1} d \alpha_{2}
$$

$$
\begin{aligned}
\delta U_{1}= & \int_{\alpha_{1} \alpha_{2}} N_{1} A_{2}\left(\frac{\partial \delta u_{0}}{\partial \alpha_{1}}+\frac{\delta v_{0}}{A_{2}} \frac{\partial A_{1}}{\partial \alpha_{2}}+\frac{A_{1} \delta w_{0}}{R_{1}}\right) d \alpha_{1} d \alpha_{2} \\
& +\int_{\alpha_{1} \alpha_{2}} M_{1} A_{2}\left(\frac{\partial \delta \theta_{1}}{\partial \alpha_{1}}+\frac{\delta \theta_{2}}{A_{2}} \frac{\partial A_{1}}{\partial \alpha_{2}}+\frac{A_{1} \delta \theta_{3}}{R_{1}}\right) d \alpha_{1} d \alpha_{2} \\
& +\int_{\alpha_{1} \alpha_{2}} N_{1}^{*} A_{2}\left(\frac{\partial \delta u_{0}^{*}}{\partial \alpha_{1}}+\frac{\delta v_{0}^{*}}{A_{2}} \frac{\partial A_{1}}{\partial \alpha_{2}}+\frac{A_{1} \delta w_{0}^{*}}{R_{1}}\right) d \alpha_{1} d \alpha_{2} \\
& +\int_{\alpha_{1} \alpha_{2}} M_{1}^{*} A_{2}\left(\frac{\partial \delta \theta_{1}^{*}}{\partial \alpha_{1}}+\frac{\delta \theta_{2}^{*}}{A_{2}} \frac{\partial A_{1}}{\partial \alpha_{2}}+\frac{A_{1} \delta \theta_{3}^{*}}{R_{1}}\right) d \alpha_{1} d \alpha_{2}
\end{aligned}
$$

In the similar way other terms can be written as:

$$
\begin{aligned}
& \delta U_{2}=\iint_{\alpha_{1} \alpha_{2}} N_{2} A_{1}\left(\frac{\partial \delta v_{0}}{\partial \alpha_{2}}+\frac{\delta u_{0}}{A_{1}} \frac{\partial A_{2}}{\partial \alpha_{1}}+\frac{A_{2} \delta w_{0}}{R_{2}}\right) d \alpha_{1} d \alpha_{2} \\
& +\iint_{\alpha_{1}} M_{2} M_{2} A_{1}\left(\frac{\partial \delta \theta_{2}}{\partial \alpha_{2}}+\frac{\delta \theta_{1}}{A_{1}} \frac{\partial A_{2}}{\partial \alpha_{1}}+\frac{A_{2} \delta \theta_{3}}{R_{2}}\right) d \alpha_{1} d \alpha_{2}
\end{aligned}
$$




$$
\begin{gathered}
+\iint_{\alpha_{1} \alpha_{2}} M_{2}^{*} A_{1}\left(\frac{\partial \delta \theta_{2}^{*}}{\partial \alpha_{2}}+\frac{\delta \theta_{1}^{*}}{A_{1}} \frac{\partial A_{2}}{\partial \alpha_{1}}+\frac{A_{2} \delta \theta_{3}^{*}}{R_{2}}\right) d \alpha_{1} d \alpha_{2} \\
\delta U_{3}=\int_{\alpha_{1} \alpha_{2}} A\left(\delta \theta_{3}\right) A_{1} A_{2} d \alpha_{1} d \alpha_{2} \\
+\int_{\alpha_{1} \alpha_{2}} 2 B\left(\delta w_{0}^{*}\right) A_{1} A_{2} d \alpha_{1} d \alpha_{2} \\
+\int_{\alpha_{1} \alpha_{2}} 3 D\left(\delta \theta_{3}^{*}\right) A_{1} A_{2} d \alpha_{1} d \alpha_{2}
\end{gathered}
$$$$
+\iint_{\alpha_{1} \alpha_{2}} P_{21}^{*} A_{1}\left(\frac{\partial \delta u_{0}^{*}}{\partial \alpha_{2}}-\frac{\delta v_{0}^{*}}{A_{1}} \frac{\partial A_{2}}{\partial \alpha_{1}}\right) d \alpha_{1} d \alpha_{2}
$$

$\delta U_{12}=\iint_{\alpha_{1} \alpha_{2}} \int_{z}\left(\tau_{12} \delta \gamma_{12}\right) A_{1} A_{2}\left(1+\frac{z}{R_{1}}\right)\left(1+\frac{z}{R_{2}}\right) d \alpha_{1} d \alpha_{2} d z$

$$
\delta U_{13}=\int_{\alpha_{1} \alpha_{2}} Q_{1} A_{2}\left(\frac{\partial \delta w_{0}}{\partial \alpha_{1}}+A_{1} \delta \theta_{1}-\frac{\delta u_{0} A_{1}}{R_{1}}\right) d \alpha_{1} d \alpha_{2}
$$

$$
+\iint_{\alpha_{1} \alpha_{2}} C_{21}^{*} A_{1}\left(\frac{\partial \delta \theta_{1}^{*}}{\partial \alpha_{2}}-\frac{\delta \theta_{2}^{*}}{A_{1}} \frac{\partial A_{2}}{\partial \alpha_{1}}\right) d \alpha_{1} d \alpha_{2}
$$

$$
\delta U_{12}=\int_{\alpha_{1}} \int_{\alpha_{2}} \int_{z}\left(\tau_{12} \delta\left(\gamma_{12}^{0}+z \chi_{12}+z^{2} \gamma_{12}^{*}+z^{3} \chi_{12}^{*}\right)\right) A_{2}
$$$$
+\int_{\alpha_{1}} \int_{\alpha_{2}} T_{1}^{*} A_{2}\left(\frac{\partial \delta w_{0}^{*}}{\partial \alpha_{1}}+3\left(A_{1} \delta \theta_{1}^{*}\right)+\frac{\delta u_{0}^{*} A_{1}}{R_{1}}\right) d \alpha_{1} d \alpha_{2}
$$

$$
\left(1+\frac{z}{R_{2}}\right) d \alpha_{1} d \alpha_{2} d z
$$

$\delta U_{12}=\iint_{\alpha_{1} \alpha_{2}} N_{12} A_{2}\left(\frac{\partial \delta v_{0}}{\partial \alpha_{1}}-\frac{\delta u_{0}}{A_{2}} \frac{\partial A_{1}}{\partial \alpha_{2}}\right) d \alpha_{1} d \alpha_{2}$

$$
+\iint_{\alpha_{1} \alpha_{2}} J_{1}^{*} A_{2}\left(\frac{\partial \delta \theta_{3}^{*}}{\partial \alpha_{1}}+2\left(\frac{\delta \theta_{1}^{*} A_{1}}{R_{1}}\right)\right) d \alpha_{1} d \alpha_{2}
$$

$$
+\iint_{\alpha_{1} \alpha_{2}} M_{12} A_{2}\left(\frac{\partial \delta \theta_{2}}{\partial \alpha_{1}}-\frac{\delta \theta_{1}}{A_{2}} \frac{\partial A_{1}}{\partial \alpha_{2}}\right) d \alpha_{1} d \alpha_{2}
$$

$$
\delta U_{23}=\iint_{\alpha_{1} \alpha_{2}} Q_{2} A_{1}\left(\frac{\partial \delta w_{0}}{\partial \alpha_{2}}+A_{2} \delta \theta_{2}-\frac{\delta v_{0} A_{2}}{R_{2}}\right) d \alpha_{1} d \alpha_{2}
$$$$
+\int_{\alpha_{1}} \int_{\alpha_{2}} S_{2} A_{1}\left(\frac{\partial \delta \theta_{3}}{\partial \alpha_{2}}+2\left(A_{2} \delta v_{0}^{*}\right)\right) d \alpha_{1} d \alpha_{2}
$$

$+\iint_{\alpha_{1} \alpha_{2}} P_{12}^{*} A_{2}\left(\frac{\partial \delta v_{0}^{*}}{\partial \alpha_{1}}-\frac{\delta u_{0}^{*}}{A_{2}} \frac{\partial A_{1}}{\partial \alpha_{2}}\right) d \alpha_{1} d \alpha_{2}$

$$
+\iint_{\alpha_{1} \alpha_{2}} T_{2}^{*} A_{1}\left(\frac{\partial \delta w_{0}^{*}}{\partial \alpha_{2}}+3\left(A_{2} \delta \theta_{2}^{*}\right)+\frac{\delta v_{0}^{*} A_{2}}{R_{2}}\right) d \alpha_{1} d \alpha_{2}
$$

$$
+\iint_{\alpha_{1} \alpha_{2}} J_{2}^{*} A_{1}\left(\frac{\partial \delta \theta_{3}^{*}}{\partial \alpha_{2}}+2\left(\frac{\delta \theta_{2}^{*} A_{2}}{R_{2}}\right)\right) d \alpha_{1} d \alpha_{2}
$$

\section{So,}

$$
\begin{aligned}
+\iint_{\alpha_{1} \alpha_{2}} N_{21} A_{1}\left(\frac{\partial \delta u_{0}}{\partial \alpha_{2}}-\frac{\delta v_{0}}{A_{1}} \frac{\partial A_{2}}{\partial \alpha_{1}}\right) d \alpha_{1} d \alpha_{2} \\
\quad+\int_{\alpha_{1} \alpha_{2}} M_{21} A_{1}\left(\frac{\partial \delta \theta_{1}}{\partial \alpha_{2}}-\frac{\delta \theta_{2}}{A_{1}} \frac{\partial A_{2}}{\partial \alpha_{1}}\right) d \alpha_{1} d \alpha_{2}
\end{aligned}
$$$$
\delta U=\delta U_{1}+\delta U_{2}+\delta U_{3}+\delta U_{12}+\delta U_{13}+\delta U_{23}
$$

$$
\delta U=\int_{\alpha_{1} \alpha_{2}} N_{1} A_{2}\left(\frac{\partial \delta u_{0}}{\partial \alpha_{1}}+\frac{\delta v_{0}}{A_{2}} \frac{\partial A_{1}}{\partial \alpha_{2}}+\frac{A_{1} \delta w_{0}}{R_{1}}\right) d \alpha_{1} d \alpha_{2}
$$




$$
\begin{aligned}
& +\iint_{\alpha_{1} \alpha_{2}} M_{1} A_{2}\left(\frac{\partial \delta \theta_{1}}{\partial \alpha_{1}}+\frac{\delta \theta_{2}}{A_{2}} \frac{\partial A_{1}}{\partial \alpha_{2}}+\frac{A_{1} \delta \theta_{3}}{R_{1}}\right) d \alpha_{1} d \alpha_{2} \\
& +\int_{\alpha_{1}} \int_{\alpha_{2}} P_{1}^{*} A_{2}\left(\frac{\partial \delta u_{0}^{*}}{\partial \alpha_{1}}+\frac{\delta v_{0}^{*}}{A_{2}} \frac{\partial A_{1}}{\partial \alpha_{2}}+\frac{A_{1} \delta w_{0}^{*}}{R_{1}}\right) d \alpha_{1} d \alpha_{2} \\
& +\int_{\alpha_{1} \alpha_{2}} \int_{1} C_{1}^{*} A_{2}\left(\frac{\partial \delta \theta_{1}}{\partial \alpha_{1}}+\frac{\delta \theta_{2}}{A_{2}} \frac{\partial A_{1}}{\partial \alpha_{2}}+\frac{A_{1} \delta \theta_{3}}{R_{1}}\right) d \alpha_{1} d \alpha_{2} \\
& +\int_{\alpha_{1} \alpha_{2}} N_{2} A_{1}\left(\frac{\partial \delta v_{0}}{\partial \alpha_{2}}+\frac{\delta u_{0}}{A_{1}} \frac{\partial A_{2}}{\partial \alpha_{1}}+\frac{A_{2} \delta w_{0}}{R_{2}}\right) d \alpha_{1} d \alpha_{2} \\
& +\iint_{\alpha_{1} \alpha_{2}} M_{2} A_{1}\left(\frac{\partial \delta \theta_{2}}{\partial \alpha_{2}}+\frac{\delta \theta_{1}}{A_{1}} \frac{\partial A_{2}}{\partial \alpha_{1}}+\frac{A_{2} \delta \theta_{3}}{R_{2}}\right) d \alpha_{1} d \alpha_{2} \\
& +\int_{\alpha_{1} \alpha_{2}} A\left(\delta \theta_{3}\right) A_{1} A_{2} d \alpha_{1} d \alpha_{2} \\
& +\int_{\alpha_{1} \alpha_{2}} 2 B\left(\delta w_{0}^{*}\right) A_{1} A_{2} d \alpha_{1} d \alpha_{2} \\
& +\int_{\alpha_{1} \alpha_{2}} 3 D\left(\delta \theta_{3}^{*}\right) A_{1} A_{2} d \alpha_{1} d \alpha_{2} \\
& +\iint_{\alpha_{1} \alpha_{2}} N_{12} A_{2}\left(\frac{\partial \delta v_{0}}{\partial \alpha_{1}}-\frac{\delta u_{0}}{A_{2}} \frac{\partial A_{1}}{\partial \alpha_{2}}\right) d \alpha_{1} d \alpha_{2} \\
& +\iint_{\alpha_{1} \alpha_{2}} M_{12} A_{2}\left(\frac{\partial \delta \theta_{2}}{\partial \alpha_{1}}-\frac{\delta \theta_{1}}{A_{2}} \frac{\partial A_{1}}{\partial \alpha_{2}}\right) d \alpha_{1} d \alpha_{2} \\
& +\int_{\alpha_{1}} \int_{\alpha_{2}} P_{12}^{*} A_{2}\left(\frac{\partial \delta v_{0}^{*}}{\partial \alpha_{1}}-\frac{\delta u_{0}^{*}}{A_{2}} \frac{\partial A_{1}}{\partial \alpha_{2}}\right) d \alpha_{1} d \alpha_{2} \\
& +\iint_{\alpha_{1} \alpha_{2}} C_{12}^{*} A_{2}\left(\frac{\partial \delta \theta_{2}^{*}}{\partial \alpha_{1}}-\frac{\delta \theta_{1}^{*}}{A_{2}} \frac{\partial A_{1}}{\partial \alpha_{2}}\right) d \alpha_{1} d \alpha_{2} \\
& +\iint_{\alpha_{1}} \int_{2} N_{21} A_{1}\left(\frac{\partial \delta u_{0}}{\partial \alpha_{2}}-\frac{\delta v_{0}}{A_{1}} \frac{\partial A_{2}}{\partial \alpha_{1}}\right) d \alpha_{1} d \alpha_{2}
\end{aligned}
$$

$$
\begin{aligned}
& +\iint_{\alpha_{1} \alpha_{2}} M_{21} A_{1}\left(\frac{\partial \delta \theta_{1}}{\partial \alpha_{2}}-\frac{\delta \theta_{2}}{A_{1}} \frac{\partial A_{2}}{\partial \alpha_{1}}\right) d \alpha_{1} d \alpha_{2} \\
& +\iint_{\alpha_{1}} N_{2} N_{21} A_{1}\left(\frac{\partial \delta u_{0}}{\partial \alpha_{2}}-\frac{\delta v_{0}}{A_{1}} \frac{\partial A_{2}}{\partial \alpha_{1}}\right) d \alpha_{1} d \alpha_{2} \\
& +\int_{\alpha_{1}} \int_{\alpha_{2}} M_{21} A_{1}\left(\frac{\partial \delta \theta_{1}}{\partial \alpha_{2}}-\frac{\delta \theta_{2}}{A_{1}} \frac{\partial A_{2}}{\partial \alpha_{1}}\right) d \alpha_{1} d \alpha_{2} \\
& \iint_{\alpha_{1}} \alpha_{2} Q_{1} A_{2}\left(\frac{\partial \delta w_{0}}{\partial \alpha_{1}}+A_{1} \delta \theta_{1}-\frac{\delta u_{0} A_{1}}{R_{1}}\right) d \alpha_{1} d \alpha_{2} \\
& +\int_{\alpha_{1} \alpha_{2}} S_{1} A_{2}\left(\frac{\partial \delta \theta_{3}}{\partial \alpha_{1}}+2\left(A_{1} \delta u_{0}^{*}\right)\right) d \alpha_{1} d \alpha_{2} \\
& \iint_{\alpha_{1}}{\alpha_{2}}_{1} P_{1}^{*} A_{2}\left(\frac{\partial \delta w_{0}^{*}}{\partial \alpha_{1}}+3\left(A_{1} \delta \theta_{1}^{*}\right)+\frac{\delta u_{0}^{*} A_{1}}{R_{1}}\right) d \alpha_{1} d \alpha_{2} \\
& +\int_{\alpha_{1} \alpha_{2}} \int_{1}^{*} A_{2}\left(\frac{\partial \delta \theta_{3}^{*}}{\partial \alpha_{1}}+2\left(\frac{\delta \theta_{1}^{*} A_{1}}{R_{1}}\right)\right) d \alpha_{1} d \alpha_{2} \\
& +\int_{\alpha_{1}} \int_{\alpha_{2}} Q_{2} A_{1}\left(\frac{\partial \delta w_{0}}{\partial \alpha_{2}}+A_{2} \delta \theta_{2}-\frac{\delta v_{0} A_{2}}{R_{2}}\right) d \alpha_{1} d \alpha_{2} \\
& +\iint_{\alpha_{1} \alpha_{2}} S_{2} A_{1}\left(\frac{\partial \delta \theta_{3}}{\partial \alpha_{2}}+2\left(A_{2} \delta v_{0}^{*}\right)\right) d \alpha_{1} d \alpha_{2} \\
& +\iint_{\alpha_{1}} Q_{2} Q_{2}^{*} A_{1}\left(\frac{\partial \delta w_{0}^{*}}{\partial \alpha_{2}}+3\left(A_{2} \delta \theta_{2}^{*}\right)+\frac{\delta v_{0}^{*} A_{2}}{R_{2}}\right) d \alpha_{1} d \alpha_{2} \\
& +\iint_{\alpha_{1}} S_{2} S_{2}^{*} A_{1}\left(\frac{\partial \delta \theta_{3}^{*}}{\partial \alpha_{2}}+2\left(\frac{\delta \theta_{2}^{*} A_{2}}{R_{2}}\right)\right) d \alpha_{1} d \alpha_{2}
\end{aligned}
$$

Now in order to eliminate derivative terms of middle surface parameters, we use integration by parts. Integrating given derivative function with respect to one of the curvilinear coordinate direction simplify the above expression. For example, let us take the first term: 


$$
\begin{gathered}
\iint_{\alpha_{1} \alpha_{2}} N_{1} A_{2}\left(\frac{\partial \delta u_{0}}{\partial \alpha_{1}}\right) d \alpha_{1} d \alpha_{2} \\
=\iint_{\alpha_{1} \alpha_{2}} \frac{\partial}{\partial \alpha_{1}}\left(N_{1} A_{2} \delta u_{0}\right) d \alpha_{1} d \alpha_{2} \\
-\iint_{\alpha_{1} \alpha_{2}}\left(\frac{\partial\left(N_{1} A_{2}\right)}{\partial \alpha_{1}}\right) \delta u_{0} d \alpha_{1} d \alpha_{2} \\
\int_{\alpha_{1} \alpha_{2}} N_{1} A_{2}\left(\frac{\partial \delta u_{0}}{\partial \alpha_{1}}\right) d \alpha_{1} d \alpha_{2} \\
=\int_{\alpha_{2}} N_{1} A_{2} \delta u_{0} d \alpha_{2}-\int_{\alpha_{1} \alpha_{2}} \int\left(\frac{\partial\left(N_{1} A_{2}\right)}{\partial \alpha_{1}}\right) \delta u_{0} d \alpha_{1} d \alpha_{2}
\end{gathered}
$$

Using the similar approach for the above expression of variation of Strain Energy one can yield following set of expression:

$$
\begin{aligned}
& \delta U=\int_{\alpha_{2}}\left(N_{1} \delta u_{0}+M_{1} \delta \theta_{1}+N_{1}^{*} \delta u_{0}^{*}+M_{1}^{*} \delta \theta_{1}^{*}\right) A_{2} d \alpha_{2} \\
& +\int_{\alpha_{2}}\left(N_{12} \delta v_{0}+M_{12} \delta \theta_{2}+N_{12}^{*} \delta v_{0}^{*}+M_{12}^{*} \delta \theta_{2}^{*}\right) A_{2} d \alpha_{2} \\
& +\int_{\alpha_{2}}\left(Q_{1} \delta w_{0}+S_{1} \delta \theta_{2}+Q_{1}^{*} \delta w_{0}^{*}+S_{1}^{*} \delta \theta_{3}^{*}\right) A_{2} d \alpha_{2} \\
& +\int_{\alpha_{1}}\left(N_{2} \delta v_{0}+M_{2} \delta \theta_{2}+N_{2}^{*} \delta v_{0}^{*}+M_{2}^{*} \delta \theta_{2}^{*}\right) A_{1} d \alpha_{1} \\
& +\int_{\alpha_{1}}\left(N_{21} \delta u_{0}+M_{21} \delta \theta_{1}+N_{21}^{*} \delta u_{0}^{*}+M_{21}^{*} \delta \theta_{1}^{*}\right) A_{1} d \alpha_{1} \\
& +\int_{\alpha_{1}}\left(Q_{2} \delta w_{0}+S_{2} \delta \theta_{3}+Q_{2}^{*} \delta w_{0}^{*}+S_{2}^{*} \delta \theta_{3}^{*}\right) A_{1} d \alpha_{1} \\
& -\int_{\alpha_{1}} \int_{\alpha_{2}}\left(\frac{\partial\left(N_{1} A_{2}\right)}{\partial \alpha_{1}}+N_{12} \frac{\partial A_{1}}{\partial \alpha_{2}}+\frac{\partial\left(N_{21} A_{1}\right)}{\partial \alpha_{2}}-N_{2} \frac{\partial A_{2}}{\partial \alpha_{1}}+Q_{1} \frac{A_{1} A_{2}}{R_{1}}\right) \\
& \delta u_{0} d \alpha_{1} d \alpha_{2}- \\
& \iint_{\alpha_{1}} \int_{\alpha_{2}}\left(\frac{\partial\left(S_{1} A_{2}\right)}{\partial \alpha_{1}}+\frac{\partial\left(S_{2} A_{1}\right)}{\partial \alpha_{2}}-\frac{M_{1} A_{1} A_{2}}{R_{1}}-\frac{M_{2} A_{1} A_{2}}{R_{2}}-A A_{1} A_{2}\right) \delta \\
& \theta_{3} d \alpha_{1} d \alpha_{2}- \\
& \int_{\alpha_{1} \alpha_{2}}\left(\frac{\partial\left(N_{1}^{*} A_{2}\right)}{\partial \alpha_{1}}+N_{12}^{*} \frac{\partial A_{1}}{\partial \alpha_{2}}+\frac{\partial\left(N_{21}^{*} A_{1}\right)}{\partial \alpha_{2}}-N_{2}^{*} \frac{\partial A_{2}}{\partial \alpha_{1}}-Q_{1}^{*} \frac{A_{1} A_{2}}{R_{1}}-2 S_{1} A_{1} A_{2}\right) \delta \\
& u_{0}^{*} d \alpha_{1} d \alpha_{2}- \\
& \int_{\alpha_{1} \alpha_{2}}\left(\frac{\partial\left(N_{12}^{*} A_{2}\right)}{\partial \alpha_{1}}+N_{21}^{*} \frac{\partial A_{2}}{\partial \alpha_{1}}+\frac{\partial\left(N_{2}^{*} A_{1}\right)}{\partial \alpha_{2}}-N_{1}^{*} \frac{\partial A_{1}}{\partial \alpha_{2}}-Q_{2}^{*} \frac{A_{1} A_{2}}{R_{1}}-2 S_{2} A_{1} A_{2}\right) \delta \\
& v_{0}^{*} d \alpha_{1} d \alpha_{2}- \\
& \int_{\alpha_{1} \alpha_{2}} \int_{(}\left(\frac{\partial\left(Q_{1}^{*} A_{2}\right)}{\partial \alpha_{1}}+\frac{\partial\left(Q_{2}^{*} A_{1}\right)}{\partial \alpha_{2}}-\frac{N_{1}^{*} A_{1} A_{2}}{R_{1}}-\frac{N_{2}^{*} A_{1} A_{2}}{R_{2}}-2 B A_{1} A_{2}\right) \delta \\
& w_{0}^{*} d \alpha_{1} d \alpha_{2}- \\
& \int_{\alpha_{1} \alpha_{2}}\left(\frac{\partial\left(M_{1}^{*} A_{2}\right)}{\partial \alpha_{1}}+M_{12}^{*} \frac{\partial A_{1}}{\partial \alpha_{2}}+\frac{\partial\left(M_{21}^{*} A_{1}\right)}{\partial \alpha_{2}}-M_{2}^{*} \frac{\partial A_{2}}{\partial \alpha_{1}}-2 S_{1}^{*} \frac{A_{1} A_{2}}{R_{1}}-3 Q_{1}^{*} A_{1} A_{2}\right) \delta
\end{aligned}
$$

$$
\begin{gathered}
\iint_{\alpha_{1}}\left(\frac{\partial\left(N_{12} A_{2}\right)}{\partial \alpha_{1}}+N_{21} \frac{\partial A_{2}}{\partial \alpha_{1}}+\frac{\partial\left(N_{2} A_{1}\right)}{\partial \alpha_{2}}-N_{1} \frac{\partial A_{1}}{\partial \alpha_{2}}+Q_{2} \frac{A_{1} A_{2}}{R_{2}}\right) \\
\delta v_{0} d \alpha_{1} d \alpha_{2}- \\
\iint_{\alpha_{1}}\left(\frac{\partial\left(Q_{1} A_{2}\right)}{\partial \alpha_{1}}+\frac{\partial\left(Q_{2} A_{1}\right)}{\partial \alpha_{2}}-\frac{N_{1} A_{1} A_{2}}{R_{1}}-\frac{N_{2} A_{1} A_{2}}{R_{2}}\right) \delta \\
\int_{\alpha_{1} \alpha_{2}}\left(\frac{\partial\left(M_{1} A_{2}\right)}{\partial \alpha_{1}}+M_{12} \frac{\partial A_{1}}{\partial \alpha_{2}}+\frac{\partial\left(M_{21} A_{1}\right)}{\partial \alpha_{2}}-M_{2} \frac{\partial A_{2}}{\partial \alpha_{1}}-Q_{1} A_{1} A_{2}\right) \delta \\
\theta_{1} d \alpha_{1} d \alpha_{2}- \\
\int_{\alpha_{1}} \int_{\alpha_{2}}\left(\frac{\partial\left(M_{12} A_{2}\right)}{\partial \alpha_{1}}+M_{21} \frac{\partial A_{2}}{\partial \alpha_{1}}+\frac{\partial\left(M_{2} A_{1}\right)}{\partial \alpha_{2}}-M_{1} \frac{\partial A_{1}}{\partial \alpha_{2}}-Q_{2} A_{1} A_{2}\right) \delta
\end{gathered}
$$$$
\theta_{2} d \alpha_{1} d \alpha_{2}-
$$ 


$$
\begin{gathered}
\theta_{1}^{*} d \alpha_{1} d \alpha_{2}- \\
\iint_{\alpha_{1} \alpha_{2}}\left(\frac{\partial\left(M_{12}^{*} A_{2}\right)}{\partial \alpha_{1}}+M_{21}^{*} \frac{\partial A_{2}}{\partial \alpha_{1}}+\frac{\partial\left(M_{2}^{*} A_{1}\right)}{\partial \alpha_{2}}-M_{1}^{*} \frac{\partial A_{1}}{\partial \alpha_{2}}-2 S_{2}^{*} \frac{A_{1} A_{2}}{R_{1}}-3 Q_{2}^{*} A_{1} A_{2}\right) \delta \\
\theta_{2}^{*} d \alpha_{1} d \alpha_{2}- \\
\iint_{\alpha_{1} \alpha_{2}}\left(\frac{\partial\left(S_{1}^{*} A_{2}\right)}{\partial \alpha_{1}}+\frac{\partial\left(S_{2}^{*} A_{1}\right)}{\partial \alpha_{2}}-\frac{M_{1}^{*} A_{1} A_{2}}{R_{1}}-\frac{M_{2}^{*} A_{1} A_{2}}{R_{2}}-3 D A_{1} A_{2}\right) \delta \\
\theta_{3}^{*} d \alpha_{1} d \alpha_{2}
\end{gathered}
$$

\section{Kinetic Energy}

Kinetic Energy term is given by:

$$
\begin{gathered}
K=\frac{1}{2} \int_{V} \rho \dot{U} \cdot \dot{U} d V \\
K=\frac{1}{2} \int_{V} \rho \dot{u} \cdot \dot{u} d V+\frac{1}{2} \int_{V} \rho \dot{v} \cdot \dot{v} d V+\frac{1}{2} \int_{V} \rho \dot{w} \cdot \dot{w} d V \\
K=\frac{1}{2} \int_{V} \rho \dot{u} \cdot \dot{u} d V+\frac{1}{2} \int_{V} \rho \dot{v} \cdot \dot{v} d V+\frac{1}{2} \int_{V} \rho \dot{w} \cdot \dot{w} d V \\
\delta K=\delta \\
K_{u}=\frac{1}{2} \int_{\alpha_{1}} \int_{\alpha_{2}} \int_{z} \rho(\dot{u})^{2}\left(1+\frac{z}{R_{1}}\right)\left(1+\frac{z}{R_{2}}\right) A_{1} A_{2} d \alpha_{1} d \alpha_{2} d z
\end{gathered}
$$

Where,

$$
\begin{aligned}
& \left(1+\frac{z}{R_{1}}\right)\left(1+\frac{z}{R_{2}}\right)=1+\frac{z^{2}}{R_{1} R_{2}}+z\left(\frac{1}{R_{1}}+\frac{1}{R_{2}}\right)(35) \\
& \dot{u}=\left(\dot{u_{0}}+z \dot{\theta_{1}}+z^{2} \cdot \dot{u_{0}^{*}}+z^{3} \dot{\theta}_{1}^{*}\right) \\
& K_{u}=\frac{1}{2} \iint_{\alpha_{1} \alpha_{2} z} \rho(\dot{u})^{2}\left\{1+\frac{z^{2}}{R_{1} R_{2}}+z\left(\frac{1}{R_{1}}+\frac{1}{R_{2}}\right)\right\}
\end{aligned}
$$

$A_{1} A_{2} d \alpha_{1} d \alpha_{2} d z$

$$
\delta K_{u}=\iint_{\alpha_{1}} \int_{\alpha_{2}} \rho
$$$$
\left(\dot{u_{0}} \delta \dot{u_{0}}+z^{2} \dot{\theta_{1}} \delta \dot{\theta_{1}}+z^{6} \dot{\theta}_{1}^{*} \delta \dot{\theta}_{1}^{*}+z \dot{u_{0}} \delta \dot{\theta_{1}}+z \dot{\theta_{1}} \delta \dot{u_{0}}\right)
$$$$
\left\{1+\frac{z^{2}}{R_{1} R_{2}}+z\left(\frac{1}{R_{1}}+\frac{1}{R_{2}}\right)\right\} A_{1} A_{2} d \alpha_{1} d \alpha_{2} d z
$$

$$
+\iint_{\alpha_{1}} \int_{\alpha_{2}} \rho
$$

$$
\begin{aligned}
& \left(z^{2} \dot{u_{0}} \delta \dot{u_{0}^{*}}+z^{2} \dot{u_{0}^{*}} \delta \dot{u_{0}}+z^{3} \dot{\theta_{1}} \delta \dot{u_{0}^{*}}+z^{3} \dot{u}_{0}^{*} \delta \dot{\theta_{1}}+z^{3} \dot{u_{0}} \delta \dot{\theta_{1}^{*}}+z^{3} \dot{\theta}_{1}^{*} \delta \dot{u_{0}}\right) \\
& \left\{1+\frac{z^{2}}{R_{1} R_{2}}+z\left(\frac{1}{R_{1}}+\frac{1}{R_{2}}\right)\right\} A_{1} A_{2} d \alpha_{1} d \alpha_{2} d z \\
& +\iint_{\alpha_{1}} \int_{\alpha_{2}} \rho \\
& \left(z^{4} \dot{u_{0}^{*}} \delta \dot{u_{0}^{*}}+z^{4} \dot{\theta_{1}} \delta \dot{\theta_{1}^{*}}+z^{4} \theta_{1}^{*} \delta \dot{\theta_{1}}+z^{5} u_{0}^{*} \delta \dot{\theta_{1}^{*}}+z^{5} \theta_{1}^{*} \delta \dot{u_{0}^{*}}\right) \\
& \left\{1+\frac{z^{2}}{R_{1} R_{2}}+z\left(\frac{1}{R_{1}}+\frac{1}{R_{2}}\right)\right\} A_{1} A_{2} d \alpha_{1} d \alpha_{2} d z \\
& \int_{t_{0}}^{t_{1}} \delta K_{u} d t=-\int_{\alpha_{1}} \int_{\alpha_{2}} \int_{z} \int_{t_{0}}^{t_{1}} \rho\left(\ddot{u_{0}}+z \ddot{\theta_{1}}+z^{2} \ddot{u_{0}^{*}}+z^{3} \ddot{\theta_{1}^{*}}\right)
\end{aligned}
$$

$$
\delta u_{0}\left\{1+\frac{z^{2}}{R_{1} R_{2}}+z\left(\frac{1}{R_{1}}+\frac{1}{R_{2}}\right)\right\} A_{1} A_{2} d \alpha_{1} d \alpha_{2} d z d t
$$$$
-\iint_{\alpha_{1}} \int_{\alpha_{2}} \int_{t_{0}}^{t_{1}} \rho\left(z \ddot{u_{0}}+z^{2} \ddot{\theta_{1}}+z^{3} \ddot{u_{0}^{*}}+z^{4} \ddot{\theta_{1}^{*}}\right) \delta \theta_{1}
$$$$
\left\{1+\frac{z^{2}}{R_{1} R_{2}}+z\left(\frac{1}{R_{1}}+\frac{1}{R_{2}}\right)\right\} A_{1} A_{2} d \alpha_{1} d \alpha_{2} d z d t
$$ 


$$
\begin{aligned}
& -\iint_{\alpha_{1} \alpha_{2}} \int_{z} \int_{t_{0}}^{t_{1}} \rho\left(z^{2} \ddot{u_{0}}+z^{3} \ddot{\theta_{1}}+z^{4} \ddot{u_{0}^{*}}+z^{5} \ddot{\theta_{1}^{*}}\right) \delta u_{0}^{*} \\
& \left\{1+\frac{z^{2}}{R_{1} R_{2}}+z\left(\frac{1}{R_{1}}+\frac{1}{R_{2}}\right)\right\} A_{1} A_{2} d \alpha_{1} d \alpha_{2} d z d t \\
& -\iint_{\alpha_{1} \alpha_{2}} \int_{z} \int_{t_{0}}^{t_{1}} \rho\left(z^{3} \ddot{u_{0}}+z^{4} \ddot{\theta_{1}}+z^{5} \ddot{u_{0}^{*}}+z^{6} \ddot{\theta_{1}^{*}}\right) \delta \theta_{1}^{*} \\
& \left\{1+\frac{z^{2}}{R_{1} R_{2}}+z\left(\frac{1}{R_{1}}+\frac{1}{R_{2}}\right)\right\} A_{1} A_{2} d \alpha_{1} d \alpha_{2} d z d t
\end{aligned}
$$

Also, we can write:

$$
\begin{aligned}
& \int_{t_{0}}^{t_{1}} \delta K_{v} d t=-\iint_{\alpha_{1} \alpha_{2}} \int_{z} \int_{t_{0}}^{t_{1}} \rho\left(\ddot{v_{0}}+z \ddot{\theta_{2}}+z^{2} \ddot{v_{0}^{*}}+z^{3} \ddot{\theta_{2}^{*}}\right) \delta v_{0} \\
& \left\{1+\frac{z^{2}}{R_{1} R_{2}}+z\left(\frac{1}{R_{1}}+\frac{1}{R_{2}}\right)\right\} A_{1} A_{2} d \alpha_{1} d \alpha_{2} d z d t \\
& -\iint_{\alpha_{1} \alpha_{2}} \int_{z} \int_{t_{0}}^{t_{1}} \rho\left(\ddot{z v_{0}}+z^{2} \ddot{\theta_{2}}+z^{3} \ddot{v_{0}^{*}}+z^{4} \ddot{\theta}_{2}^{*}\right) \delta \theta_{2} \\
& \left\{1+\frac{z^{2}}{R_{1} R_{2}}+z\left(\frac{1}{R_{1}}+\frac{1}{R_{2}}\right)\right\} A_{1} A_{2} d \alpha_{1} d \alpha_{2} d z d t
\end{aligned}
$$$$
-\iint_{\alpha_{1}} \int_{\alpha_{2}} \int_{z}^{t_{1}} \rho\left(z_{t_{0}}^{2} \underline{v}_{0}+z^{3} \ddot{\theta_{2}}+z^{4} \ddot{v_{0}^{*}}+z^{5} \ddot{\theta}_{2}^{*}\right) \delta v_{0}^{*}
$$$$
\left\{1+\frac{z^{2}}{R_{1} R_{2}}+z\left(\frac{1}{R_{1}}+\frac{1}{R_{2}}\right)\right\} A_{1} A_{2} d \alpha_{1} d \alpha_{2} d z d t
$$$$
-\iint_{\alpha_{1}} \int_{\alpha_{2}} \int_{z}^{t_{1}} \rho\left(z^{3} \ddot{v_{0}}+z^{4} \ddot{\theta_{2}}+z^{5} \ddot{v_{0}^{*}}+z^{6} \ddot{\theta}_{2}^{*}\right) \delta \theta_{2}^{*}
$$$$
\left\{1+\frac{z^{2}}{R_{1} R_{2}}+z\left(\frac{1}{R_{1}}+\frac{1}{R_{2}}\right)\right\} A_{1} A_{2} d \alpha_{1} d \alpha_{2} d z d t
$$

$$
\begin{aligned}
& \int_{t_{0}}^{t_{1}} \delta K_{w} d t=-\iint_{\alpha_{1} \alpha_{2}} \iint_{t_{0}}^{t_{1}} \rho\left(\ddot{w_{0}}+z \ddot{\theta_{3}}+z^{2} \ddot{w_{0}^{*}}+z^{3} \ddot{\theta}_{3}^{*}\right) \delta w_{0} \\
& \left\{1+\frac{z^{2}}{R_{1} R_{2}}+z\left(\frac{1}{R_{1}}+\frac{1}{R_{2}}\right)\right\} A_{1} A_{2} d \alpha_{1} d \alpha_{2} d z d t \\
& -\iint_{\alpha_{1} \alpha_{2}} \int_{z} \int_{t_{1}}^{t_{1}} \rho\left(\ddot{w_{0}}+z^{2} \ddot{\theta_{3}}+z^{3} \ddot{w_{0}^{*}}+z^{4} \ddot{\theta}_{3}^{*}\right) \delta \theta_{3} \\
& \left\{1+\frac{z^{2}}{R_{1} R_{2}}+z\left(\frac{1}{R_{1}}+\frac{1}{R_{2}}\right)\right\} A_{1} A_{2} d \alpha_{1} d \alpha_{2} d z d t \\
& -\iint_{\alpha_{1} \alpha_{2}} \int_{z}^{t_{t_{1}}} \rho\left(z^{2} w_{0}+z^{3} \ddot{\theta_{3}}+z^{4} \ddot{w_{0}^{*}}+z^{5} \ddot{\theta}_{3}^{*}\right) \delta w_{0}^{*} \\
& \left\{1+\frac{z^{2}}{R_{1} R_{2}}+z\left(\frac{1}{R_{1}}+\frac{1}{R_{2}}\right)\right\} A_{1} A_{2} d \alpha_{1} d \alpha_{2} d z d t \\
& \left\{\int_{\alpha_{1} \alpha_{2} z} \iint_{t_{0}}^{t_{1}} \rho\left(z^{3} \ddot{w_{0}}+z^{4} \ddot{\theta_{3}}+z^{5} \ddot{w_{0}^{*}}+z^{6} \ddot{\theta_{3}^{*}}\right) \delta \theta_{3}^{*}\right. \\
& \left\{\frac{z^{2}}{R_{1} R_{2}}+z\left(\frac{1}{R_{1}}+\frac{1}{R_{2}}\right)\right\} A_{1} A_{2} d \alpha_{1} d \alpha_{2} d z d t
\end{aligned}
$$

Above three set of equations (38), (39) \& (40) are part of the dynamic forces produced because of displacement function along the ' $\alpha_{1}$ ' curvilinear coordinate, ' $\alpha_{2}$ ' curvilinear coordinate and surface normal direction ' $z$ ' respectively. Here, without taking any assumptions, all inertia forces (i.e. rotatory inertia, translational inertia) have been accounted. Also, it is to be noted that this formulation is valid for theory assuming $\left(\frac{h}{R_{\text {min }}}\right)^{2}<<1$. In case of theory with assumption $\left(\frac{h}{R_{\min }}\right) \ll<1$ term from the equation (35) will turn into unity. 


\section{Equations of Static Equilibrium}

Substituting the terms derived for variation in external work, internal strain energy and edge stresses, we can expand the principle of minimum total potential energy as given in equation (7b). Further the variation form of equation can be obtained corresponding to the small change in displacement parameters. Also this variational expression contains stress resultants across edges which can be given as boundary conditions associated with the problem. Following are the set of equilibrium equations for static problem for HOSNT12 model adopted for doubly curved shell.

$$
\begin{aligned}
\delta u_{0}: & \frac{\partial\left(N_{1} A_{2}\right)}{\partial \alpha_{1}}+N_{12} \frac{\partial A_{1}}{\partial \alpha_{2}}+\frac{\partial\left(N_{21} A_{1}\right)}{\partial \alpha_{2}} \\
& -N_{2} \frac{\partial A_{2}}{\partial \alpha_{1}}+Q_{1} \frac{A_{1} A_{2}}{R_{1}}+q_{1} A_{1} A_{2}=0 \\
\delta v_{0}: & \frac{\partial\left(N_{12} A_{2}\right)}{\partial \alpha_{1}}+N_{21} \frac{\partial A_{2}}{\partial \alpha_{1}}+\frac{\partial\left(N_{2} A_{1}\right)}{\partial \alpha_{2}} \\
& -N_{1} \frac{\partial A_{1}}{\partial \alpha_{2}}+Q_{2} \frac{A_{1} A_{2}}{R_{2}}+q_{2} A_{1} A_{2}=0 \\
\delta w_{0}: & \frac{\partial\left(Q_{1} A_{2}\right)}{\partial \alpha_{1}}+\frac{\partial\left(Q_{2} A_{1}\right)}{\partial \alpha_{2}}-\frac{N_{1} A_{1} A_{2}}{R_{1}} \\
\delta \theta_{2}: & \frac{\partial\left(M_{12} A_{2}\right)}{\partial \alpha_{1}}+M_{21} \frac{\partial A_{2}}{\partial \alpha_{1}}+\frac{\partial\left(M_{2} A_{1}\right)}{\partial \alpha_{2}} \\
& -\frac{N_{2} A_{1} A_{2}}{R_{2}}-q_{n} A_{1} A_{2}=0 \\
\delta \theta_{1}: & \frac{\partial\left(M_{1} A_{2}\right)}{\partial \alpha_{1}}+M_{12} \frac{\partial A_{1}}{\partial \alpha_{2}}+\frac{\partial\left(M_{21} A_{1}\right)}{\partial \alpha_{2}}-Q_{2} A_{1} A_{2}+m_{2} A_{1} A_{2}=0 \\
& -M_{2} \frac{\partial A_{2}}{\partial \alpha_{1}}-Q_{1} A_{1} A_{2}+m_{1} A_{1} A_{2}=0 \\
&
\end{aligned}
$$

$$
\begin{aligned}
\delta \theta_{3}: & \frac{\partial\left(S_{1} A_{2}\right)}{\partial \alpha_{1}}+\frac{\partial\left(S_{2} A_{1}\right)}{\partial \alpha_{2}}-\frac{M_{1} A_{1} A_{2}}{R_{1}} \\
& -\frac{M_{2} A_{1} A_{2}}{R_{2}}-A A_{1} A_{2}-m_{n} A_{1} A_{2}=0
\end{aligned}
$$$$
\delta u_{0}^{*}: \frac{\partial\left(N_{1}^{*} A_{2}\right)}{\partial \alpha_{1}}+N_{12}^{*} \frac{\partial A_{1}}{\partial \alpha_{2}}+\frac{\partial\left(N_{21}^{*} A_{1}\right)}{\partial \alpha_{2}}
$$$$
-N_{2}^{*} \frac{\partial A_{2}}{\partial \alpha_{1}}-Q_{1}^{*} \frac{A_{1} A_{2}}{R_{1}}-2 S_{1} A_{1} A_{2}+\frac{h^{2}}{4}\left(q_{1}\right) A_{1} A_{2}=0
$$$$
\delta v_{0}^{*}: \frac{\partial\left(N_{12}^{*} A_{2}\right)}{\partial \alpha_{1}}+N_{21}^{*} \frac{\partial A_{2}}{\partial \alpha_{1}}+\frac{\partial\left(N_{2}^{*} A_{1}\right)}{\partial \alpha_{2}}
$$$$
-N_{1}^{*} \frac{\partial A_{1}}{\partial \alpha_{2}}-Q_{2}^{*} \frac{A_{1} A_{2}}{R_{1}}-2 S_{2} A_{1} A_{2}+\frac{h^{2}}{4}\left(q_{2}\right) A_{1} A_{2}=0
$$$$
\delta w_{0}^{*}: \frac{\partial\left(Q_{1}^{*} A_{2}\right)}{\partial \alpha_{1}}+\frac{\partial\left(Q_{2}^{*} A_{1}\right)}{\partial \alpha_{2}}-\frac{N_{1}^{*} A_{1} A_{2}}{R_{1}}
$$$$
-\frac{N_{2}^{*} A_{1} A_{2}}{R_{2}}-2 B A_{1} A_{2}-\frac{h^{2}}{4}\left(q_{n}\right) A_{1} A_{2}=0
$$

$$
\delta \theta_{1}^{*}: \frac{\partial\left(M_{1}^{*} A_{2}\right)}{\partial \alpha_{1}}+M_{12}^{*} \frac{\partial A_{1}}{\partial \alpha_{2}}+\frac{\partial\left(M_{21}^{*} A_{1}\right)}{\partial \alpha_{2}}
$$$$
-M_{2}^{*} \frac{\partial A_{2}}{\partial \alpha_{1}}-2 S_{1}^{*} \frac{A_{1} A_{2}}{R_{1}}-3 Q_{1}^{*} A_{1} A_{2}+\frac{h^{2}}{4}\left(m_{1}\right) A_{1} A_{2}=0
$$

$$
\delta \theta_{2}^{*}: \frac{\partial\left(M_{12}^{*} A_{2}\right)}{\partial \alpha_{1}}+M_{21}^{*} \frac{\partial A_{2}}{\partial \alpha_{1}}+\frac{\partial\left(M_{2}^{*} A_{1}\right)}{\partial \alpha_{2}}
$$$$
-M_{1}^{*} \frac{\partial A_{1}}{\partial \alpha_{2}}-2 S_{2}^{*} \frac{A_{1} A_{2}}{R_{1}}-3 Q_{2}^{*} A_{1} A_{2}+\frac{h^{2}}{4}\left(m_{2}\right) A_{1} A_{2}=0
$$

$\delta \theta_{3}^{*}: \frac{\partial\left(S_{1}^{*} A_{2}\right)}{\partial \alpha_{1}}+\frac{\partial\left(S_{2}^{*} A_{1}\right)}{\partial \alpha_{2}}-\frac{M_{1}^{*} A_{1} A_{2}}{R_{1}}$ 


$$
-\frac{M_{2}^{*} A_{1} A_{2}}{R_{2}}-3 D A_{1} A_{2}-\frac{h^{2}}{4}\left(m_{n}\right) A_{1} A_{2}=0
$$

Boundary conditions can be given for edges:

$\alpha_{1}=$ constant edge:

$$
\begin{array}{r}
N_{1}=\overline{N_{1}} \text { or } u_{0}=\overline{u_{0}} \quad N_{12}=\overline{N_{12}} \text { or } v_{0}^{*}=\overline{v_{0}^{*}} \\
Q_{1}^{*}=\overline{Q_{1}^{*}} \text { or } w_{0}^{*}=\overline{w_{0}^{*}}
\end{array}
$$

$M_{1}=\overline{M_{1}}$ or $\theta_{1}=\overline{\theta_{1}} \quad M_{12}=\overline{M_{12}}$ or $\theta_{2}=\overline{\theta_{2}}$

$$
S_{1}^{*}=\overline{S_{1}^{*}} \text { or } \theta_{3}=\overline{\theta_{3}}
$$

$$
N_{1}^{*}=\overline{N_{1}^{*}} \text { or } u_{0}^{*}=\overline{u_{0}^{*}} \quad N_{12}^{*}=\overline{N_{12}^{*}} \text { or } v_{0}^{*}=\overline{v_{0}^{*}}
$$$$
Q_{1}^{*}=\overline{Q_{1}^{*}} \text { or } w_{0}^{*}=\overline{w_{0}^{*}}
$$

$$
M_{1}^{*}=\overline{M_{1}^{*}} \text { or } \theta_{1}^{*}=\overline{\theta_{1}^{*}} M_{12}^{*}=\overline{M_{12}^{*}} \text { or } \theta_{2}^{*}=\overline{\theta_{2}^{*}}
$$

$$
S_{1}^{*}=\overline{S_{1}^{*}} \text { or } \theta_{2}^{*}=\overline{\theta_{2}^{*}}
$$

$\alpha_{2}=$ constant edge:

$$
N_{21}=\overline{N_{21}} \text { or } u_{0}=\overline{u_{0}} \quad M_{21}^{*}=\overline{M_{21}^{*}} \text { or } \theta_{1}^{*}=\overline{\theta_{1}^{*}}
$$

$$
\begin{array}{cc}
M_{21}=\overline{M_{21}} \text { or } \theta_{1}=\overline{\theta_{1}} & N_{2}=\overline{N_{2}} \text { or } v_{0}=\overline{v_{0}} \\
N_{21}^{*}=\overline{N_{21}^{*}} \text { or } u_{0}^{*}=\overline{u_{0}^{*}} & M_{2}=\overline{M_{2}} \text { or } \theta_{2}=\overline{\theta_{2}} \\
N_{2}^{*}=\overline{N_{2}^{*}} \text { or } v_{0}^{*}=\overline{v_{0}^{*}} \quad Q_{2}=\overline{Q_{2}} \text { or } w_{0}=\overline{w_{0}} \\
S_{2}^{*}=\overline{S_{2}^{*}} \text { or } \theta_{2}^{*}=\overline{\theta_{2}^{*}} \\
M_{2}^{*}=\overline{M_{2}^{*}} \text { or } \theta_{2}^{*}=\overline{\theta_{2}^{*}} \quad S_{2}=\overline{S_{2}} \text { or } \theta_{3}=\overline{\theta_{3}} \\
Q_{2}^{*}=\overline{Q_{2}^{*}} \text { or } w_{0}^{*}=\overline{w_{0}^{*}}
\end{array}
$$

\section{Equations of Dynamic Equilibrium}

Taking account of Hamilton's principle and substituting expression for kinetic energy we get equations of dynamic equilibrium. Following set of twelve expressions for HOSNT12 model are derived corresponding to each displacement parameter. These terms are added in the above set of static equilibrium equations to represent equations of dynamic equilibrium.

Contribution of kinetic energy terms with respect to middle surface parameters for HOSNT12 with $\left(\frac{h}{R_{\text {min }}}\right)^{2} \ll 1$ can be given as:

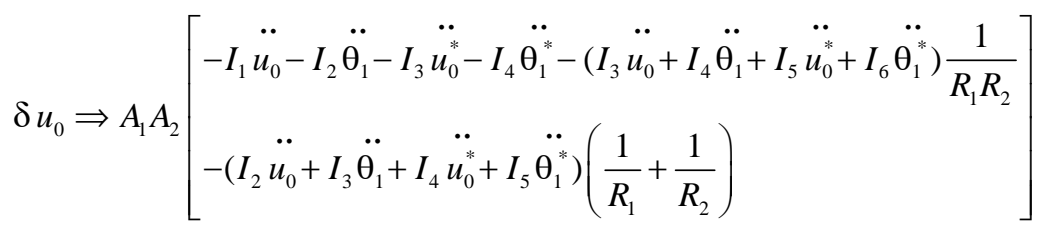

$$
\begin{aligned}
& \delta v_{0} \Rightarrow A_{1} A_{2}\left[\begin{array}{l}
\ddot{\ddot{v_{0}}}-I_{2} \ddot{\theta_{2}}-I_{3} \ddot{v_{0}^{*}}-I_{4} \ddot{\theta_{2}^{*}}-\left(I_{3} \ddot{v_{0}}+I_{4} \ddot{\theta_{2}}+I_{5} \ddot{v_{0}^{*}}+I_{6} \ddot{\theta_{2}^{*}}\right) \frac{1}{R_{1} R_{2}} \\
-\left(I_{2} \ddot{v_{0}}+I_{3} \ddot{\theta_{2}}+I_{4} \ddot{v_{0}^{*}}+I_{5} \ddot{\theta_{2}^{*}}\right)\left(\frac{1}{R_{1}}+\frac{1}{R_{2}}\right)
\end{array}\right] \\
& \delta w_{0} \Rightarrow A_{1} A_{2}\left[\begin{array}{l}
-I_{1} \ddot{w_{0}}-I_{2} \ddot{\theta_{3}}-I_{3} \ddot{w_{0}^{*}}-I_{4} \ddot{\theta_{3}^{*}}-\left(I_{3} \ddot{w_{0}}+I_{4} \ddot{\theta_{3}}+I_{5} \ddot{w_{0}^{*}}+I_{6} \ddot{\theta}_{3}^{*}\right) \frac{1}{R_{1} R_{2}} \\
-\left(I_{2} \ddot{w_{0}}+I_{3} \ddot{\theta_{3}}+I_{4} \ddot{w_{0}^{*}}+I_{5} \ddot{\theta_{3}^{*}}\right)\left(\frac{1}{R_{1}}+\frac{1}{R_{2}}\right)
\end{array}\right]
\end{aligned}
$$




$$
\begin{aligned}
& \delta \theta_{1} \Rightarrow A_{1} A_{2}\left[\begin{array}{l}
-I_{2} \ddot{u_{0}}-I_{3} \ddot{\theta_{1}}-I_{4} \ddot{u_{0}^{*}}-I_{5} \ddot{\theta_{1}^{*}}-\left(I_{4} \ddot{u_{0}}+I_{5} \ddot{\theta_{1}}+I_{6} \ddot{u_{0}^{*}}+I_{7} \ddot{\theta}_{1}^{*}\right) \frac{1}{R_{1} R_{2}} \\
-\left(I_{3} \ddot{u_{0}}+I_{4} \ddot{\theta_{1}}+I_{5} \ddot{u_{0}^{*}}+I_{6} \ddot{\theta_{1}^{*}}\right)\left(\frac{1}{R_{1}}+\frac{1}{R_{2}}\right)
\end{array}\right] \\
& \delta \theta_{2} \Rightarrow A_{1} A_{2}\left[\begin{array}{l}
\ddot{-} \ddot{v_{0}}-I_{3} \ddot{\theta_{2}}-I_{4} \ddot{v_{0}^{*}}-I_{5} \ddot{\theta_{2}^{*}}-\left(I_{4} \ddot{v_{0}}+I_{5} \ddot{\theta_{2}}+I_{6} \ddot{v_{0}^{*}}+I_{7} \ddot{\theta_{2}^{*}}\right) \frac{1}{R_{1} R_{2}} \\
-\left(I_{3} \overrightarrow{v_{0}}+I_{4} \ddot{\theta_{2}}+I_{5} \ddot{v_{0}^{*}}+I_{6} \ddot{\theta_{2}^{*}}\right)\left(\frac{1}{R_{1}}+\frac{1}{R_{2}}\right)
\end{array}\right] \\
& \delta \theta_{3} \Rightarrow A_{1} A_{2}\left[\begin{array}{l}
-I_{2} \ddot{w_{0}}-I_{3} \ddot{\theta_{3}}-I_{4} \ddot{w_{0}^{*}}-I_{5} \ddot{\theta_{3}^{*}}-\left(I_{4} \ddot{w_{0}}+I_{5} \ddot{\theta_{3}}+I_{6} \ddot{w_{0}^{*}}+I_{7} \ddot{\theta}_{3}^{*}\right) \frac{1}{R_{1} R_{2}} \\
-\left(I_{3} \ddot{w_{0}}+I_{4} \ddot{\theta_{3}}+I_{5} \ddot{w_{0}^{*}}+I_{6} \ddot{\theta_{3}^{*}}\right)\left(\frac{1}{R_{1}}+\frac{1}{R_{2}}\right)
\end{array}\right]
\end{aligned}
$$

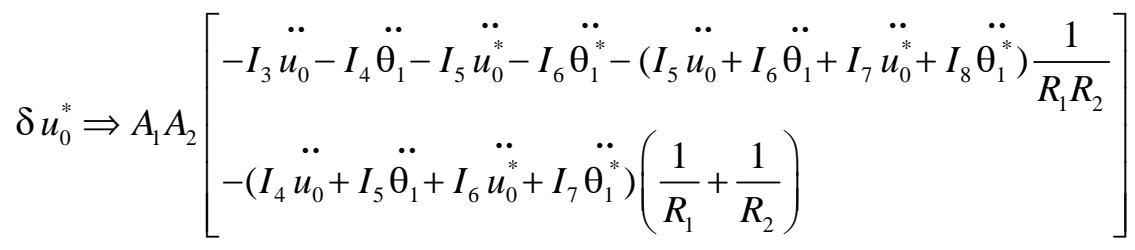

$$
\begin{aligned}
& \delta v_{0}^{*} \Rightarrow A_{1} A_{2}\left[\begin{array}{l}
-I_{3} \ddot{v_{0}}-I_{4} \ddot{\theta_{2}}-I_{5} \ddot{v_{0}^{*}}-I_{6} \ddot{\theta_{2}^{*}}-\left(I_{5} \ddot{v_{0}}+I_{6} \ddot{\theta_{2}}+I_{7} \ddot{v_{0}^{*}}+I_{8} \ddot{\theta_{2}^{*}}\right) \frac{1}{R_{1} R_{2}} \\
-\left(I_{4} \ddot{v_{0}}+I_{5} \ddot{\theta_{2}}+I_{6} \ddot{v_{0}^{*}}+I_{7} \ddot{\theta_{2}^{*}}\right)\left(\frac{1}{R_{1}}+\frac{1}{R_{2}}\right)
\end{array}\right]
\end{aligned}
$$

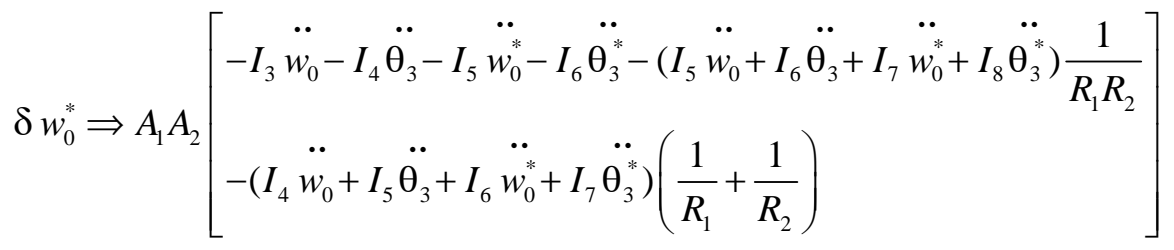

$$
\begin{aligned}
& \delta \theta_{1}^{*} \Rightarrow A_{1} A_{2}\left[\begin{array}{l}
-I_{4} \ddot{u_{0}}-I_{5} \ddot{\theta_{1}}-I_{6} \ddot{u_{0}^{*}}-I_{7} \ddot{\theta_{1}^{*}}-\left(I_{6} \ddot{u_{0}}+I_{7} \ddot{\theta_{1}}+I_{8} \ddot{u_{0}^{*}}+I_{9} \ddot{\theta}_{1}^{*}\right) \frac{1}{R_{1} R_{2}} \\
-\left(I_{5} \ddot{u_{0}}+I_{6} \ddot{\theta_{1}}+I_{7} \ddot{u_{0}^{*}}+I_{8} \ddot{\theta_{1}^{*}}\right)\left(\frac{1}{R_{1}}+\frac{1}{R_{2}}\right)
\end{array}\right]
\end{aligned}
$$




$$
\begin{aligned}
& \delta \theta_{2}^{*} \Rightarrow A_{1} A_{2}\left[\begin{array}{l}
-I_{4} \ddot{v_{0}}-I_{5} \ddot{\theta_{2}}-I_{6} \ddot{v_{0}^{*}}-I_{7} \ddot{\theta_{2}^{*}}-\left(I_{6} \ddot{v_{0}}+I_{7} \ddot{\theta_{2}}+I_{8} \ddot{v_{0}^{*}}+I_{9} \ddot{\theta_{2}^{*}}\right) \frac{1}{R_{1} R_{2}} \\
-\left(I_{5} \ddot{v_{0}}+I_{6} \ddot{\theta_{2}}+I_{7} \ddot{v_{0}^{*}}+I_{8} \ddot{\theta_{2}^{*}}\right)\left(\frac{1}{R_{1}}+\frac{1}{R_{2}}\right)
\end{array}\right] \\
& \delta \theta_{3}^{*} \Rightarrow A_{1} A_{2}\left[\begin{array}{l}
-I_{4} \ddot{w_{0}}-I_{5} \ddot{\theta_{3}}-I_{6} \ddot{w_{0}^{*}}-I_{7} \ddot{\theta_{3}^{*}}-\left(I_{6} \ddot{w_{0}}+I_{7} \ddot{\theta_{3}}+I_{8} \ddot{w}_{0}^{*}+I_{9} \ddot{\theta_{3}^{*}}\right) \frac{1}{R_{1} R_{2}} \\
-\left(I_{5} \ddot{w_{0}}+I_{6} \ddot{\theta_{3}}+I_{7} \ddot{w_{0}^{*}}+I_{8} \ddot{\theta_{3}^{*}}\right)\left(\frac{1}{R_{1}}+\frac{1}{R_{2}}\right)
\end{array}\right]
\end{aligned}
$$

Contribution of kinetic energy terms with respect to middle surface parameters for HOSNT12 with $\left(\frac{h}{R_{\min }}\right) \ll<$ can be given as:

$$
\begin{aligned}
& \delta u_{0} \Rightarrow A_{1} A_{2}\left[-I_{1} \ddot{u_{0}}-I_{2} \ddot{\theta_{1}}-I_{3} \ddot{u_{0}^{*}}-I_{4} \ddot{\theta_{1}^{*}}\right] \\
& \delta v_{0} \Rightarrow A_{1} A_{2}\left[-I_{1} \ddot{v_{0}}-I_{2} \ddot{\theta_{2}}-I_{3} \ddot{v_{0}^{*}}-I_{4} \ddot{\theta_{2}^{*}}\right] \\
& \delta w_{0} \Rightarrow A_{1} A_{2}\left[-I_{1} \ddot{w_{0}}-I_{2} \ddot{\theta_{3}}-I_{3} \ddot{w_{0}^{*}}-I_{4} \ddot{\theta_{3}^{*}}\right] \\
& \delta \theta_{1} \Rightarrow A_{1} A_{2}\left[-I_{2} \ddot{u_{0}}-I_{3} \ddot{\theta_{1}}-I_{4} \ddot{u_{0}^{*}}-I_{5} \ddot{\theta_{1}^{*}}\right] \\
& \delta \theta_{2} \Rightarrow A_{1} A_{2}\left[-I_{2} \ddot{v_{0}}-I_{3} \ddot{\theta_{2}}-I_{4} \ddot{v_{0}^{*}}-I_{5} \ddot{\theta_{2}^{*}}\right] \\
& \delta \theta_{3} \Rightarrow A_{1} A_{2}\left[-I_{2} \ddot{w_{0}}-I_{3} \ddot{\theta_{3}}-I_{4} \ddot{w_{0}^{*}}-I_{5} \ddot{\theta_{3}^{*}}\right] \\
& \delta u_{0}^{*} \Rightarrow A_{1} A_{2}\left[-I_{3} \ddot{u_{0}}-I_{4} \ddot{\theta_{1}}-I_{5} \ddot{u_{0}^{*}}-I_{6} \ddot{\theta_{1}^{*}}\right]
\end{aligned}
$$

$$
\delta v_{0}^{*} \Rightarrow A_{1} A_{2}\left[-I_{3} \ddot{v_{0}}-I_{4} \ddot{\theta_{2}}-I_{5} \ddot{v_{0}^{*}}-I_{6} \ddot{\theta_{2}^{*}}\right]
$$$$
\delta w_{0}^{*} \Rightarrow A_{1} A_{2}\left[-I_{3} \ddot{w_{0}}-I_{4} \ddot{\theta_{3}}-I_{5} \ddot{w_{0}^{*}}-I_{6} \ddot{\theta_{3}^{*}}\right]
$$$$
\delta \theta_{1}^{*} \Rightarrow A_{1} A_{2}\left[-I_{4} \ddot{u_{0}}-I_{5} \ddot{\theta_{1}}-I_{6} \ddot{u_{0}^{*}}-I_{7} \ddot{\theta_{1}^{*}}\right]
$$$$
\delta \theta_{2}^{*} \Rightarrow A_{1} A_{2}\left[-I_{4} \ddot{v_{0}}-I_{5} \ddot{\theta_{2}}-I_{6} \ddot{v_{0}^{*}}-I_{7} \ddot{\theta_{2}^{*}}\right]
$$$$
\delta \theta_{3}^{*} \Rightarrow A_{1} A_{2}\left[-I_{4} \ddot{w_{0}}-I_{5} \ddot{\theta_{3}}-I_{6} \ddot{w_{0}^{*}}-I_{7} \ddot{\theta_{3}^{*}}\right]
$$

where,

$$
\begin{aligned}
& \left\{I_{1}, I_{2}, I_{3}, I_{4}, I_{5}, I_{6}, I_{7}, I_{8}, I_{9}\right\} \\
& =\int_{-h / 2}^{h / 2} \rho(z)\left\{1, z, z^{2}, z^{3}, z^{4}, z^{5}, z^{6}, z^{7}, z^{8}\right\} d z
\end{aligned}
$$

\section{Numerical Analysis}

The general mathematical model for a doubly curved shell given above is particularized for an open cylindrical shell (Fig. 4) defined by $(x, \phi, z)$ coordinates and incorporating the following geometrical equations.

$$
\begin{aligned}
& R_{1}=\infty \quad R_{2}=R \quad A_{1} d \alpha_{1}=d x \\
& A_{2} d \alpha_{2}=R d \phi
\end{aligned}
$$




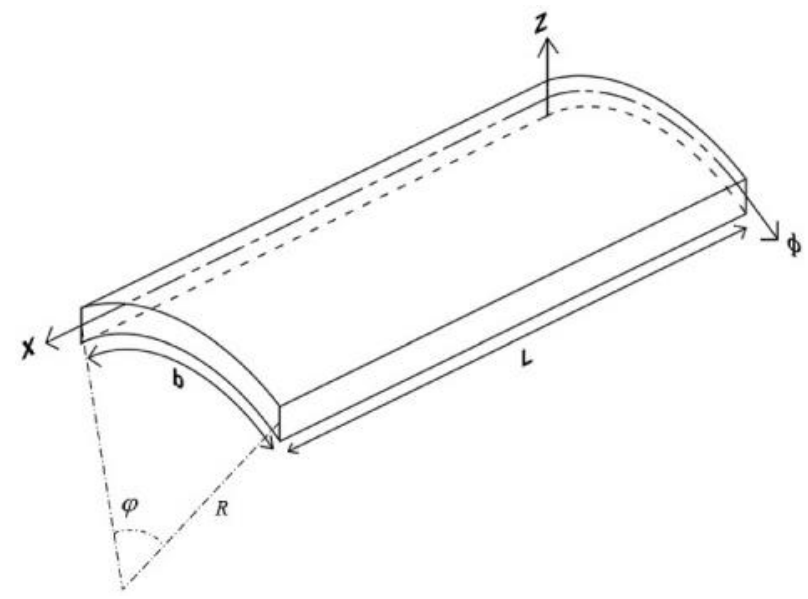

Fig. 4: Geometry of circular cylindrical shell panel points:

The above relation can be deduced to following

$$
A_{1}=1 \quad A_{2}=R \quad d \alpha_{1}=d x \quad d \alpha_{2}=d \phi
$$

Example: A circular cylindrical shell subjected to sinusoidal load $\left(q_{0} \sin \frac{\pi x}{L} \cos \frac{\pi \phi}{\varphi}\right)$ in radial direction. The material of the shell is considered to be isotropic with Poisson's ratio being 0.3. Table 1 presents results for normalized displacement and stresses for different $(R / L)$ ratios. Results as given by present HOSNT12 model for both thickness criteria show an excellent agreement with the 3D results given by Bhimaraddi and Chandrashekhara (Bhimaraddi \& Chandrashekhara 1992).

\section{Conclusions}

Present work derives the set of static and dynamic equilibrium equations using the higher order shear and normal deformation theory with twelve middle surface parameters. The set of equations are derived for a doubly curved shell structure and present theory also assumes extended thickness criteria as given by Flugge. Above derived set of equations can further be reduced to other cylindrical, spherical shells or plate structures using necessary geometric characteristic. The results presented for cylindrical shells verify the accuracy of present theory.

Table 1: Transverse displacement and stresses for diaphragm supported isotropic circular cylindrical shell under sinusoidal transverse load $(h / L=0.1 ; L / b=1)$

$$
\left(\frac{E \bar{w}}{q_{0} R}\right)_{(x, \phi, z)=(L / 2, \varphi / 2,0)}\left(\frac{\left(\overline{\sigma_{1}}\right)_{(x, \phi, z)=(L / 2, \varphi / 2, h / 2)}}{q_{0}}\right)
$$

\begin{tabular}{lcccccc} 
Theory & Exact 3D & $\begin{array}{c}\text { HOSNT12a } \\
(\% \text { Error })\end{array}$ & $\begin{array}{c}\text { HOSNT12b } \\
(\% \text { Error })\end{array}$ & $\begin{array}{c}\text { Exact 3D } \\
\text { HOSNT12a }\end{array}$ & $\begin{array}{c}\text { HOSNT12b } \\
(\% \text { Error })\end{array}$ \\
\hline$R / L=5$ & 29.003 & $28.9471(0.193)$ & $28.9276(0.26)$ & 21.138 & $21.1774(0.19)$ & $21.2461(0.51)$ \\
$R / L=10$ & 29.379 & $29.3654(0.046)$ & $29.3600(0.065)$ & 20.719 & $20.7948(0.37)$ & $20.8326(0.55)$ \\
$R / L=20$ & 29.445 & $29.4413(0.013)$ & $29.4398(0.018)$ & 20.413 & $20.5011(0.43)$ & $20.5208(0.53)$
\end{tabular}

\begin{tabular}{lcccccc} 
& & $\left(\frac{\left(\overline{\tau_{13}}\right)_{(x, \phi, z)=(0, \varphi / 2,0)}}{q_{0}}\right)$ & & $\left(\frac{\left(\overline{\sigma_{3}}\right)_{(x, \phi, z)=(L / 2, \varphi / 2, h / 2)}}{q_{0}}\right)$ & \\
$R / L=5$ & 2.3455 & $2.3436(0.081)$ & $2.3414(0.17)$ & NA & 1.2224 & 1.2332 \\
$R / L=10$ & 2.3787 & $2.3787(00)$ & $2.2781(4.23)$ & NA & 1.2261 & 1.2318 \\
$R / L=20$ & 2.3847 & $2.3852(0.021)$ & $2.3850(0.012)$ & NA & 1.2271 & 1.2300 \\
\hline
\end{tabular}

HOSNT12a: Theory with $(\mathrm{h} / \mathrm{R})^{2}<<1$, HOSNT12b: Theory with $(\mathrm{h} / \mathrm{R})<<1$ 


\section{Appendix A}

Flugge-Lur'e Byrne Criteria for $(h / R)^{2}<<1$

$$
\begin{aligned}
\frac{1}{\left(1+\frac{z}{R_{i}}\right)}= & \left(1+\frac{z}{R_{i}}\right)^{-1}=1-\frac{z}{R_{i}}+\left(\frac{z}{R_{i}}\right)^{2}-\left(\frac{z}{R_{i}}\right)^{3}+\ldots \ldots \ldots . \\
& \frac{\left(1+\frac{z}{R_{2}}\right)}{\left(1+\frac{z}{R_{1}}\right)}=\left(1+\frac{z}{R_{1}}\right)^{-1}\left(1+\frac{z}{R_{2}}\right) \\
& \frac{\left(1+\frac{z}{R_{2}}\right)}{\left(1+\frac{z}{R_{1}}\right)}=\left(1-\frac{z}{R_{1}}+\left(\frac{z}{R_{1}}\right)^{2}-\left(\frac{z}{R_{1}}\right)^{3}+\ldots \ldots \ldots\right) \\
& \frac{\left(1+\frac{z}{R_{2}}\right)}{\left(1+\frac{z}{R_{1}}\right)}=\left(1-\frac{z}{R_{1}}+\left(\frac{z}{R_{1}}\right)^{2}-\left(\frac{z}{R_{1}}\right)^{3}+\ldots \ldots \ldots . . .\right.
\end{aligned}
$$

$$
\begin{aligned}
& \frac{\left(1+\frac{z}{R_{2}}\right)}{\left(1+\frac{z}{R_{1}}\right)}=\left(1-\frac{z}{R_{1}}+\left(\frac{z}{R_{1}}\right)^{2}-\left(\frac{z}{R_{1}}\right)^{3}+\ldots \ldots \ldots\right)\left(1+\frac{z}{R_{2}}\right) \\
& \frac{\left(1+\frac{z}{R_{2}}\right)}{\left(1+\frac{z}{R_{1}}\right)}=\left(1-\left(\frac{z}{R_{1}}-\frac{z}{R_{2}}\right)+\frac{z^{2}}{R_{1}}\left(\frac{1}{R_{1}}-\frac{1}{R_{2}}\right)-\frac{z^{3}}{R_{1}^{2}}\left(\frac{1}{R_{1}}-\frac{1}{R_{2}}\right)+\frac{z^{4}}{R_{1}^{3}}\left(\frac{1}{R_{1}}-\frac{1}{R_{2}}\right)-\frac{z^{5}}{R_{1}^{4}}\left(\frac{1}{R_{1}}-\frac{1}{R_{2}}\right)+\ldots \ldots \ldots\right) \\
& \text { As we know }\left(\frac{z}{R_{i}}\right)^{2}<<1 \text {, so neglecting higher order terms. } \\
& \frac{\left(1+\frac{z}{R_{2}}\right)}{\left(1+\frac{z}{R_{1}}\right)}=\left(1-z\left(\frac{1}{R_{1}}-\frac{1}{R_{2}}\right)+\frac{z^{2}}{R_{1}}\left(\frac{1}{R_{1}}-\frac{1}{R_{2}}\right)\right)
\end{aligned}
$$


Similarly,

$$
\frac{\left(1+\frac{z}{R_{1}}\right)}{\left(1+\frac{z}{R_{2}}\right)}=\left(1-z\left(\frac{1}{R_{2}}-\frac{1}{R_{1}}\right)+\frac{z^{2}}{R_{2}}\left(\frac{1}{R_{2}}-\frac{1}{R_{1}}\right)\right)
$$

Or general expression with further multiples of thickness coordinate can be written as follows:

$$
\frac{z^{n}\left(1+\frac{z}{R_{j}}\right)}{\left(1+\frac{z}{R_{i}}\right)}=\left(z^{n}-z^{n+1}\left(\frac{1}{R_{i}}-\frac{1}{R_{j}}\right)+\frac{z^{n+2}}{R_{i}}\left(\frac{1}{R_{i}}-\frac{1}{R_{j}}\right)\right)
$$

\section{References}

Bhimaraddi A and Chandrashekhara K (1992) Three-dimensional elasticity solution for static response of simply supported orthotropic cylindrical shells Composite Structures 20227 235

Kant T (1981) A higher-order general shell theory, Rep C/R/391/ 81 , University of Wales, Swansea

Kant T (1981) A higher-order general laminated shell theory Rep $C / R / 395 / 81$, University of Wales, Swansea

Kant T (2002) Reflections on shell theories. In: Proc International Conference on Advances in Civil Engineering, IIT Kharagpur

Kant T (1976) Thick Shells of Revolution-Some Studies. Ph.D. Thesis, Indian Institute of Technology Bombay

Kraus H (1967) Thin Elastic Shells 1st ed., New York: John Wiley \& Sons, Ltd

Love AE H (1888) On the Small Free Vibrations and Deformations of Thin Elastic Shells Phil Trans Roy Soc 179 491-549. 